\title{
๖The Power Distribution between Symmetric and Antisymmetric Components of the Tropical Wavenumber-Frequency Spectrum
}

\author{
Ofer Shamir, ${ }^{\mathrm{a}}$ Chen Schwartz, ${ }^{\mathrm{a}}$ Chaim I. Garfinkel, ${ }^{\mathrm{a}}$ And Nathan Paldor ${ }^{\mathrm{a}}$ \\ ${ }^{\mathrm{a}}$ Fredy and Nadine Herrmann Institute of Earth Sciences, Hebrew University of Jerusalem, Jerusalem, Israel
}

(Manuscript received 21 September 2020, in final form 26 March 2021)

\begin{abstract}
A yet unexplained feature of the tropical wavenumber-frequency spectrum is its parity distribution, i.e., the distribution of power between the meridionally symmetric and antisymmetric components of the spectrum. Due to the linearity of the decomposition to symmetric and antisymmetric components and the Fourier analysis, the total spectral power equals the sum of the power contained in each of these two components. However, the spectral power need not be evenly distributed between the two components. Satellite observations and reanalysis data provide ample evidence that the parity distribution of the tropical wavenumber-frequency spectrum is biased toward its symmetric component. Using an intermediate-complexity model of an idealized moist atmosphere, we find that the parity distribution of the tropical spectrum is nearly insensitive to large-scale forcing, including topography, ocean heat fluxes, and land-sea contrast. On the other hand, we find that a small-scale (stochastic) forcing has the capacity to affect the parity distribution at large spatial scales via an upscale (inverse) turbulent energy cascade. These results are qualitatively explained by considering the effects of triad interactions on the parity distribution. According to the proposed mechanism, any bias in the small-scale forcing, symmetric or antisymmetric, leads to symmetric bias in the large-scale spectrum regardless of the source of variability responsible for the onset of the asymmetry. As this process is also associated with the generation of large-scale features in the tropics by small-scale convection, the present study demonstrates that the physical process associated with deep convection leads to a symmetric bias in the tropical spectrum.
\end{abstract}

KEYWORDS: Atmosphere; Tropics; Shallow-water equations; Spectral analysis/models/distribution; Asymmetry

\section{Introduction}

A staple in the study of tropical meteorology is the wavenumber-frequency spectra of satellite-derived deepconvection proxies, such as outgoing longwave radiation (OLR) and brightness temperature (BT). For example, the wavenumber-frequency spectrum obtained using BT satellite observations sourced from the Cloud Archive User Service (CLAUS) dataset is shown in Fig. 1 (see Kiladis et al. 2009). The main features of the tropical power spectrum, as indicated by this figure, are (Wheeler and Kiladis 1999; Cho et al. 2004; Masunaga et al. 2006; Hendon and Wheeler 2008; Kiladis et al. 2009) strong spectral peaks along the dispersion curves of convectively coupled equatorial waves; strong spectral peaks in the region of the wavenumber-frequency plane corresponding to the Madden-Julian oscillation (MJO); and a red background in both wavenumber and frequency, in the broad sense of a smoothly decaying signal away from the origin.

The observed wave features in Fig. 1 fit the theoretical dispersion curves of equatorial waves predicted by Matsuno (1966) remarkably well. Matsuno's solutions consist of frequencies (eigenvalues) and corresponding latitude-dependent amplitudes (eigenfunctions) of zonally propagating wave solutions of the shallow-water (SW) equations on the (unbounded)

\footnotetext{
D Denotes content that is immediately available upon publication as open access.

Corresponding author: Nathan Paldor, nathan.paldor@mail.huji. ac.il
}

equatorial $\beta$ plane. The solution space of this system corresponds to three families of solutions, each with a countable number of modes, namely, the westward-propagating inertiagravity (WIG) waves, the westward-propagating equatorial Rossby (ER) waves, and the eastward-propagating inertiagravity (EIG) waves. In addition, the solution space of the system also includes two mixed modes that fill the frequency gap between the IG waves and Rossby waves families, namely, the westward-propagating mixed Rossby-gravity (MRG) wave and the Kelvin wave (Delplace et al. 2017; Garfinkel et al. 2017; Paldor et al. 2018). In addition to the wavenumber and mode number (that determine the wave mode), the solutions of the SW equations are also determined by the layer depth in a motionless basic state. In practice, in a baroclinic atmosphere, the linearized SW equations describe the horizontal motion of each vertical mode separately. In this case, the "layer depth" is determined from the separation constant between the horizontal and vertical equations, referred to as the equivalent depth. The observed wave features in the tropical wavenumber-frequency spectrum correspond to "equivalent depths" of between 12 and $50 \mathrm{~m}$ (indicated by solid black curves in Fig. 1).

The analytic solutions of the SW equations on the equatorial $\beta$ plane have also been employed in the context of the MJO, where the Matsuno-Gill model (Matsuno 1966; Gill 1980) is used to find the transient response to an easterly moving heat source mimicking a moving convective region (Chao 1987; Biello and Majda 2005; Majda and Stechmann 2009; Sobel and Maloney 2012; Adames and Kim 2016; Kacimi and Khouider 2018). In this model, the response to a simple Gaussian (equatorially symmetric) heat source consists of meridionally 


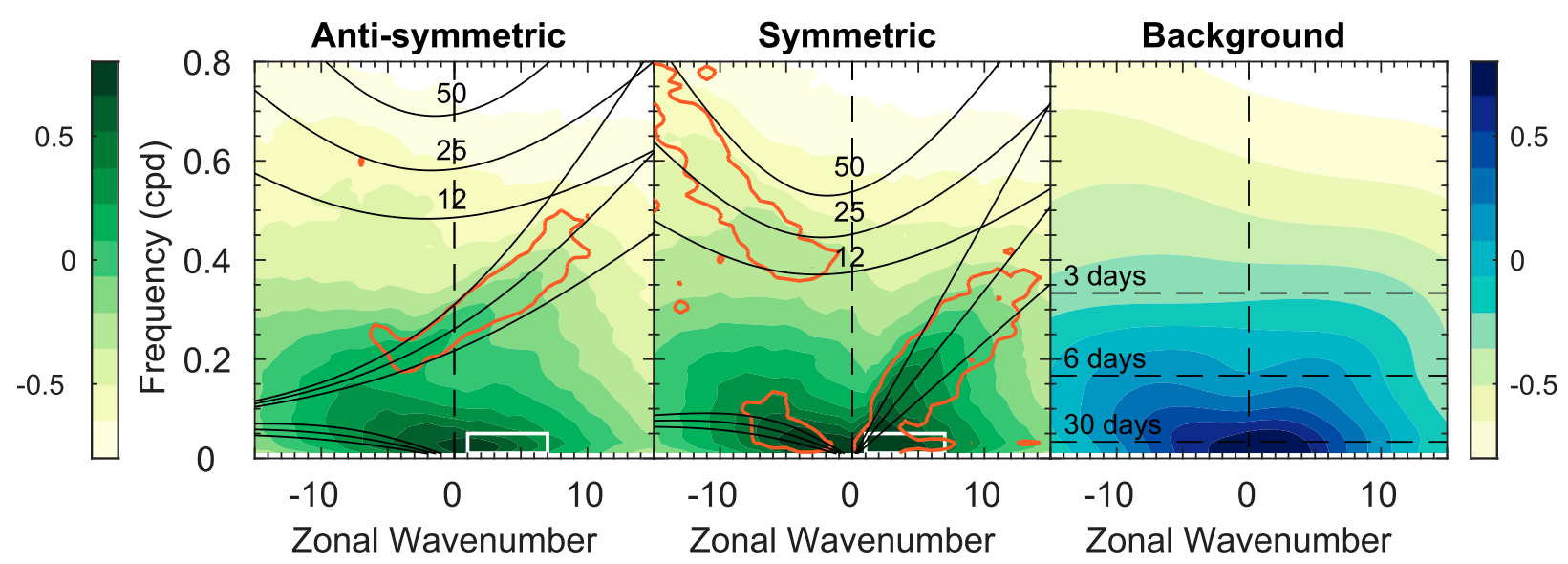

FIG. 1. Tropical wavenumber-frequency spectrum of the brightness temperature (BT). Green shading: (left) antisymmetric and (center) symmetric components of the raw power spectrum in base-10 logarithmic scale. Blue shading: (right) background spectrum, obtained from the raw spectrum by successive applications of a 1-2-1 filter in both wavenumber and frequency. The number of applied passes in frequency is 10 throughout, while the number of passes in the wavenumber varies from 10 for frequencies below $0.2 \mathrm{cpd}$, through 20 for frequencies between 0.2 and $0.3 \mathrm{cpd}$, up to 40 for frequencies above $0.3 \mathrm{cpd}$. The solid orange contour marks the level for which the raw/background equals 1.2 and the signal is statistically significant to at least the $95 \%$ level (for an estimated 100 degrees of freedom, assuming no latitudinal independence). The data are described in section 2.

symmetric Kelvin and Rossby waves, which is consistent with the stronger projection of the MJO on the symmetric part of the spectrum.

In addition to the power concentrated along the dispersion curves of the SW waves, there is substantial power evident in between these curves, which is referred to as the background spectrum. This power was postulated by Wheeler and Kiladis (1999) to be red in both wavenumber and frequency, an assumption that was explicitly used in subsequent works to calculate the background spectrum (Hendon and Wheeler 2008; Masunaga et al. 2006). However, the underlying theoretical basis for this postulate is still lacking.

In the present study we wish to explore another, yet unexplained, feature of the tropical wavenumber-frequency spectrum, which is its parity distribution, i.e., the distribution of power between the symmetric and antisymmetric parts of the spectrum. A standard practice in the above studies is to decompose the gridded (latitude-dependent) field into meridionally symmetric and antisymmetric components, e.g., $\mathrm{BT}(\phi)=$ $\operatorname{BTS}(\phi)+\operatorname{BTA}(\phi)$, where $\operatorname{BTS}(\phi)=[\mathrm{BT}(\phi)+\mathrm{BT}(-\phi)] / 2$ and $\operatorname{BTA}(\phi)=[\mathrm{BT}(\phi)-\mathrm{BT}(-\phi)] / 2$, and consider the spectrum of each of these two components separately. Due to the linearity of this decomposition and the space-time (Fourier) analysis, the spectral power at any combination of wavenumbers and frequencies, and any latitudinal band, equals the sum of the powers in BTS and BTA at the same combination of wavenumbers and frequencies and the same latitudinal band. However, the spectral power need not be evenly distributed between the symmetric and antisymmetric components. Indeed, upon closer inspection of Fig. 1, it can be observed that the symmetric component of the spectrum contains more power than the antisymmetric component. Specifically, the total power in the symmetric component summed over all wavenumbers and frequencies (of the raw spectrum) is 31\% higher than the total power in the antisymmetric component, relative to the latter. Some of this bias can be attributed to the contribution of the MJO, which is projected almost exclusively on the symmetric component. Nevertheless, even after filtering the contribution of the MJO (as indicated by the white rectangle in the figure) the total power in the symmetric component is still $26 \%$ higher than the total power in the antisymmetric component.

The first question we explore is whether the symmetric bias in Fig. 1 is a robust feature of the tropical wavenumberfrequency spectrum, or whether it is a unique feature of the BT. To this end we study in section 3 the parity distribution of the wavenumber-frequency spectra of zonal wind, meridional wind, vertical wind, geopotential height, divergence, and vorticity fields from reanalysis data in both the troposphere and stratosphere. We find that the total power in all cases is indeed biased toward the symmetric part of the spectrum, although to a different extent in each variable.

Having established the existence of a parity bias in the tropical wavenumber-frequency spectrum, the next question is, What is its origin? The approach we take in the present work for probing this question is a mechanistic approach aimed at understanding how different processes influence the parity distribution, as opposed to a phenomenological approach aimed at associating the parity distribution with particular variability sources. To this end, we study in section 4 the effects of both large- and small-scale forcing. We find that large-scale forcing, including topography, ocean heat fluxes, and landsea contrast have little effect on the parity distribution of the tropical wavenumber-frequency spectrum. On the other hand, a small-scale stochastic forcing has the capacity to influence the parity distribution of the wavenumber-frequency spectrum at large spatial scales via the process of an upscale (inverse) turbulent energy cascade. Based on these results, 
we propose, in section 5, a mechanism that can explain some of the symmetric bias using triad interactions. According to the proposed mechanism, any asymmetry (symmetric or antisymmetric) in the small-scale forcing leads to a symmetric bias in the tropical spectrum at large spatial scales, regardless of the forcing source. This provides a qualitative explanation for the higher power in the symmetric part of the spectrum. A more quantitative explanation of the particular amount of bias requires a more quantitative explanation for the onset of the asymmetries, which will ultimately require tracing back the origins of the variability.

\section{Data and methods}

Four complementary sources of data are analyzed in this study: BT satellite observations; ERA5 data, used for establishing the existence of a bias and for studying the parity distribution of the tropical wavenumber-frequency spectrum across different dynamical variables and different pressure levels; idealized general circulation model simulations, used for studying the effects of both large- and small-scale forcing on the parity distribution of the tropical wavenumber-frequency spectrum; SW model simulations, used as a lower complexity model to hone in on the relevant mechanism and eliminate sensitivities to the vertical structure of the small-scale forcing in the higher complexity model. Detailed descriptions of these four datasets are given in sections $2 \mathrm{a}-\mathrm{d}$, below. The details of the small-scale forcing are described in section 2e. The parity distribution in all datasets was analyzed using the wavenumber-frequency spectra obtained using space-time analysis as detailed in section $2 \mathrm{f}$.

\section{a. Satellite data}

Satellite data consist of uniform latitude-longitude gridded dataset of BT produced from $10 \mu \mathrm{m}$ thermal infrared radiance from the operational meteorological satellites participating in the International Satellite Cloud Climatology Project (ISCCP), described in Hodges et al. (2000) and obtained from the NASA Langley Atmospheric Sciences Data Center (LASDC). The data are taken from the CLAUS low-resolution (interpolated) dataset consisting of four times daily estimates from 1 July 1983 to 30 June 2009, with a spatial resolution of $0.5^{\circ} \times 0.5^{\circ}$.

\section{b. Reanalysis data}

To obtain the observed wavenumber-frequency spectrum across various dynamical variables, we use the new generation of the European Centre for Medium-Range Weather Forecasts (ECMWF) reanalysis data, ERA5 (Hersbach et al. 2020). The data used here were downloaded at 6-h temporal resolution between the years 1979 and 2018 at a horizontal resolution of $1.25^{\circ} \times 1.25^{\circ}$.

\section{c. Idealized model simulations}

To study the sensitivity of the parity distribution of the wavenumber-frequency spectrum to both large- and smallscale forcing we use an intermediate-complexity Model of an idealized Moist Atmosphere (MiMA), introduced by Jucker and Gerber (2017). We compare the parity distribution of the tropical wavenumber-frequency spectrum in two experiments, with and without topography, ocean heat fluxes, and land-sea contrast. The configuration of these elements follows that of Garfinkel et al. (2020b). Briefly, the ocean heat fluxes include parameterizations for the mean meridional heat flux, the Pacific and Atlantic warm pools, and the poleward directed currents - the Gulf Stream and Kuroshio. The land-sea contrast parameterizes the effects of the differences in mechanical damping, differences in evaporation, and the differences in heat capacity. For the experiment with topography, a realistic topography is used. For the experiment without topography, the topographic height over land areas is set uniformly to $15 \mathrm{~m}$. The same model is also used to study the effects of upscale (inverse) turbulent energy cascade on the parity distribution by adding the small-scale stochastic forcing described in section $2 \mathrm{e}$. The smallscale forcing is added to the model configuration with no topography, ocean heat fluxes, and land-sea contrast in order to isolate its effect from the large-scale forcing. In all cases the model is integrated for 40 years at a spectral resolution of T42, i.e., a triangular truncation where the highest retained wavenumber and total wavenumber both equal 42 , and 40 evenly spaced pressure levels. The time-space analysis is performed on the last 38 years of the integration, after a 2-yr spinup time.

\section{d. Shallow-water model simulations}

To understand the governing mechanism behind the effects of upscale energy cascade on the parity distribution, we use the framework of the forced-dissipated shallow-water equations. To this end, we use the Geophysical Fluid Dynamics Laboratory's (GFDL's) spectral-transformed shallow-water model (more details are given in https://www.gfdl.noaa.gov/ idealized-spectral-models-quickstart/). The results of section 5 were obtained using a spectral resolution of T85, a time step of $d t=600 \mathrm{~s}$, a Robert-Asselin filter coefficient of 0.04 , a Rayleigh friction coefficient of $1 / 6 \mathrm{day}^{-1}$, and the second-order $\left(\nabla^{4}\right)$ hyperdiffusion coefficient was set such that the highest resolved Fourier mode has a damping rate of 10 day $^{-1}$. The diffusion terms are added to the momentum equations. For the purpose of the statistical analysis, the model was integrated for 10000 days with a sampling rate of $6 \mathrm{~h}$ and the analysis was applied to the last 9200 days after a 2-yr spinup time.

\section{e. Small-scale stochastic forcing}

To investigate the effects of an upscale energy cascade on the parity distribution in the tropics we use the following stochastic forcing. Let $S_{m l}^{i}$ denote the spectral coefficients of the source term in a spherical harmonics decomposition, where $m$ and $l$ are the order (i.e., zonal wavenumber) and degree of the spherical harmonics, respectively (see, e.g., Boyd 2001) and $i$ is the time step, then the applied forcing is described in spectral form by

$$
S_{m l}^{i}=\left(1-e^{-2 d t / \tau}\right)^{1 / 2} Q^{i}+e^{-d t / \tau} S_{m l}^{i-1},
$$

where $d t=600 \mathrm{~s}$ is the time step, $\tau=2$ days is the decorrelation time and $Q^{i}$ is a uniformly sampled array with elements in $(-A, A)$, where $A$ is the forcing amplitude. The results of sections 4 and 5 were obtained using forcing amplitudes of $A=$ $2 \times 10^{-4}$ and $A=5 \times 10^{-4}$, respectively, which leads to a rootmean-squared velocity similar to that in ERA5 data at 500 mbar. The forcing is masked in physical space by a Gaussian in latitude 
TABLE 1. The weights applied to the odd and even modes of the stochastic forcing in spectral space in each of the 11 experiments, ranging from a purely symmetric forcing (Exp 1), through evenly distributed forcing (Exp 6) to a purely antisymmetric forcing (Exp 11). The weights are chosen so as to preserve the amount of total input spectral energy between the different experiments.

\begin{tabular}{lccccccccccc}
\hline \hline & Exp 1 & $\operatorname{Exp} 2$ & $\operatorname{Exp} 3$ & $\operatorname{Exp} 4$ & $\operatorname{Exp} 5$ & $\operatorname{Exp} 6$ & $\operatorname{Exp} 7$ & $\operatorname{Exp} 8$ & $\operatorname{Exp} 9$ & $\operatorname{Exp} 10$ & $\operatorname{Exp} 11$ \\
\hline Even weights & 1.354 & 1.285 & 1.211 & 1.133 & 1.049 & 0.957 & 0.856 & 0.742 & 0.606 & 0.428 & 0 \\
Odd weights & 0 & 0.469 & 0.663 & 0.812 & 0.938 & 1.049 & 1.149 & 1.241 & 1.327 & 1.407 & 1.483 \\
\hline
\end{tabular}

centered around the equator with an $e$-folding of $15^{\circ}$ latitudes, and in spectral space by retaining only wavenumbers $30 \leq|m| \leq 40$ and degree $30 \leq l \leq 40$. We use these values of $m$ and $l$ in order to distance the forcing from the resulting linear waves' response, which is concentrated at low wavenumbers (typically $|m| \leq 10$ ). The 2-day decorrelation time scale is similar to that used in section 6 of Salby and Garcia (1987) and is justified by observations of clouds (Orlanski and Polinsky 1977).

A similar forcing was used in Vallis et al. (2004) and Barnes et al. (2010), which in the midlatitude context mimics the stirring of the barotropic flow by baroclinic eddies. Note, Vallis et al. (2004) and Barnes et al. (2010) used this forcing in the framework of the barotropic vorticity equation to which the source term was added. In the SW model the source term was added to the continuity equation. Physically, one can think of "convection" as adding/removing mass stochastically but with some memory. We stress that the applied forcing is not representative of small-scale convection in the tropics, which has typical wavenumbers in the range $1000 \leqq|k| \lesssim 4000$. Nevertheless, it captures the physical mechanism of upscale turbulent energy cascade. The results remain qualitatively similar if the source term is added to the vorticity equation instead. In the MiMA model the source was added to the temperature equation with a sinusoidal mask in the vertical direction between 200 and 1000 mbar and zero above 200 mbar. Physically, one can think of release/uptake of latent heat.

To control the parity distribution of the applied forcing, the source term in (1) is further masked in spectral space by applying additional weights to differentiate even and odd values of $l-|m|$ (which determine the meridional symmetry). The weights were determined so as to preserve the amount of input spectral energy. For $30 \leq|m| \leq 40$ and $30 \leq l \leq 40$, with $l \geq|m|$, the stochastic forcing consists of a total of 66 modes in spectral space. Out of these 66 modes, 36 correspond to even values of $l-|m|$ and 30 to odd values. Before applying additional weight, the total spectral energy of the applied forcing is $\sum_{m=30}^{40} \sum_{l=m}^{40} 1^{2}=66$. Thus, if $w_{o}$ and $w_{e}$ denote the odd and even weights applied to the spectral forcing, then in order to conserve the amount of input spectral energy they must satisfy $30 w_{o}^{2}+36 w_{e}^{2}=66$. For example, the resulting weights for an applied forcing with evenly distributed parity are $w_{o}=1.049$ and $w_{e}=0.957$. In the present work we let the parity of the input spectral energy vary from $100 \%$ symmetric to $100 \%$ antisymmetric with all consecutive decades in between for a total of 11 experiments. The resulting weights in all 11 experiments are given in Table 1.

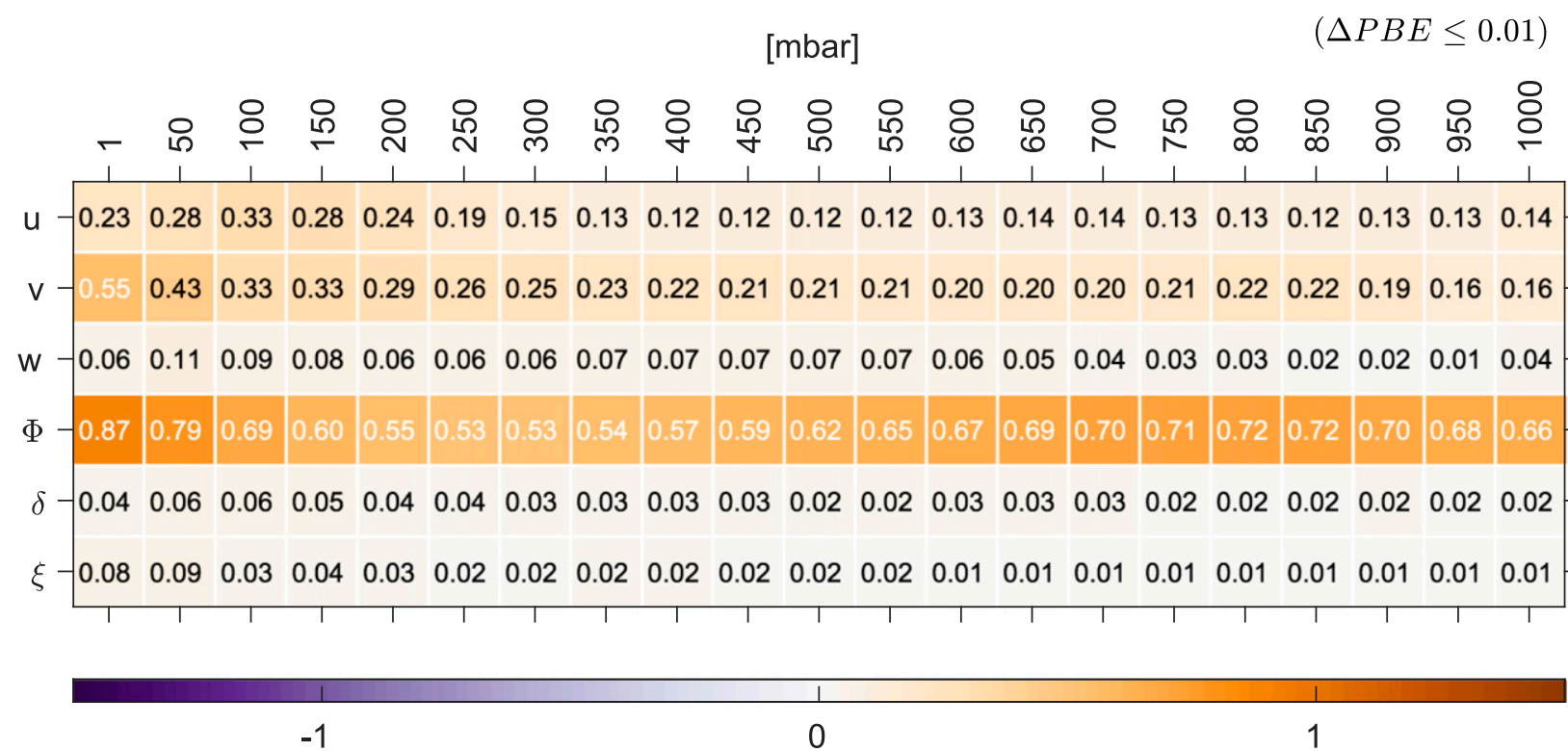

FIG. 2. The parity bias estimator (PBE), defined in Eq. (2), in the tropical wavenumber-frequency spectra of the ERA5 data for different pressure levels (columns) and each of the following dynamical variables (rows): zonal wind $(u)$, meridional wind $(v)$, vertical wind $\left(w\right.$, in Pa s $\left.{ }^{-1}\right)$, geopotential height $(\Phi)$, divergence $(\delta)$, and vorticity $(\xi)$. The PBEs in this figure were obtained using the total power in the symmetric and antisymmetric components of the wavenumber-frequency spectra in the region $|k| \leq 15$ and $\omega \leq 0.8 \mathrm{cpd}$, after filtering out the MJO contribution as indicated in Fig. 1. The uncertainties in the PBEs were estimated using a chi-squared test to obtain the confidence intervals of the variance to the $95 \%$ levels with 150 degrees of freedom. The reported value at the top-right corner corresponds to the mean uncertainty across all variables and all pressure levels. 


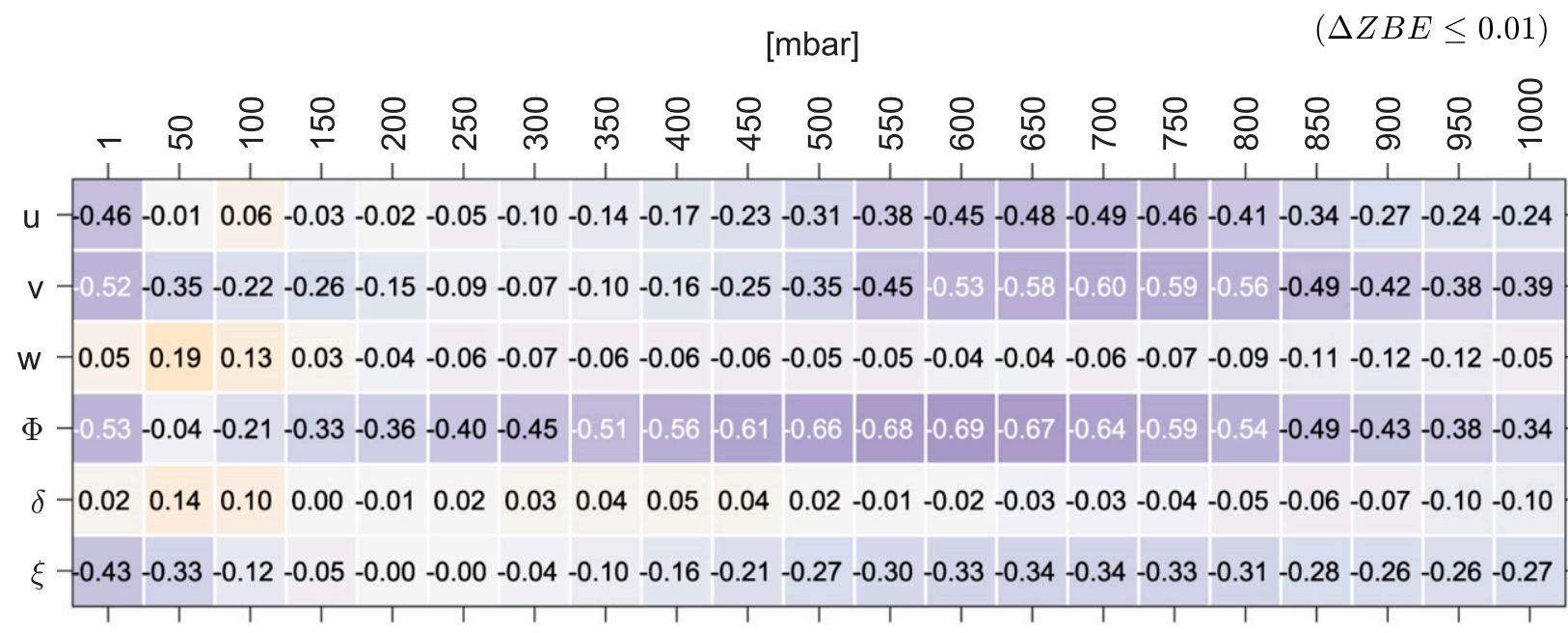

FIG. 3. The zonal bias estimator (ZBE), defined in Eq. (3), in the tropical wavenumber-frequency spectra of the ERA5 data for different pressure levels (columns) and each of the following dynamical variables (rows): zonal wind $(u)$, meridional wind ( $v$ ), vertical wind $(w$, in $\operatorname{Pa~s}^{-1}$ ), geopotential height $(\Phi)$, divergence $(\delta)$, and vorticity $(\xi)$. The ZBEs in this figure were obtained using the total power in the eastward and westward components of the wavenumber-frequency spectra in the region $|k| \leq 15$ and $\omega \leq 0.8 \mathrm{cpd}$, after filtering out the MJO contribution as indicated in Fig. 1. The uncertainties in the PBEs were estimated using a chi-square test to obtain the confidence intervals of the variance to the $95 \%$ levels with 150 degrees of freedom. The reported value at the top-right corner corresponds to the mean uncertainty across all variables and all pressure levels.

\section{f. Space-time analysis}

The power spectra throughout this work are obtained by applying a two-dimensional Fourier analysis in space and time as in Wheeler and Kiladis (1999) and Kiladis et al. (2009). To this end we used the open-source wkSpaceTime routine of the NCAR Command Language, ${ }^{1}$ which implements the analysis described in the above papers without the tropical depression filter used in the latter. The results of the following sections were obtained using a temporal window of 96 days with an overlap of 10 days between consecutive windows, and a meridional window of $15^{\circ} \mathrm{S}-15^{\circ} \mathrm{N}$. The results remain qualitatively the same for 30 day overlap between consecutive windows or for meridional windows of $10^{\circ} \mathrm{S}-10^{\circ} \mathrm{N}$ and $25^{\circ} \mathrm{S}-25^{\circ} \mathrm{N}$.

\section{Observed parity distribution in reanalysis data}

To substantiate the existence of a parity bias in the tropical wavenumber-frequency spectrum we examine, in this section, the parity distribution in the ERA5 data. Recall that we define the parity distribution as the distribution of power between the symmetric and antisymmetric components of the spectrum. After applying the space-time analysis and summing over latitude to obtain the power in the tropics, the resulting spectra

\footnotetext{
${ }^{1}$ As pointed out by a reviewer, the reader is cautioned of a bug in the wkSpaceTime routine that sums the power along the equator twice in cases where the data have an equatorial grid point.
}

depend on the dynamical variable being used for the analysis and the pressure level at which it is sampled. Thus, we consider the parity distribution as a function of the two, considering the zonal wind, meridional wind, vertical wind, geopotential height, divergence, and vorticity for the dynamical variables and a range of pressure levels extending from 1 mbar at the top of the stratosphere to 1000 mbar at the surface.

A convenient way of quantifying the parity bias is using the following estimator, referred to here as the "parity bias estimator" (PBE):

$$
\mathrm{PBE}=\frac{s-a}{s+a},
$$

where $s$ and $a$ denote the symmetric and antisymmetric parts of some feature. This PBE varies between -1 when the considered feature is purely antisymmetric to +1 when the considered feature is purely symmetric (and in particular is well defined in both cases). In addition, by dividing $s$ and $a$ by their sum, this PBE can be interpreted as the signed difference between the odds of the two complementary events.

The resulting PBEs of the ERA5 data obtained using the total power over $|k| \leq 15$ and $\omega \leq 0.8$ cpd are shown in Fig. 2 for the different pressure levels (columns) and different dynamical variables (rows). In all cases, across all pressure levels and dynamical variables, the total power is ubiquitously biased toward the symmetric part of the spectrum to some degree. To give a sense of the numerical values in Fig. 2, a PBE value of 0.01 corresponds to a symmetric bias relative to the antisymmetric component of $2 \%$, while a PBE value of $1 / 3$ already 
corresponds to a symmetric bias of $100 \%$. For comparison, the corresponding PBE of the BT in Fig. 1 (after filtering out the MJO contribution) equals $0.12( \pm 0.01)$, which corresponds to a symmetric bias relative to the antisymmetric component of $26 \%$. Note, the PBE values in this figure were obtained using the total spectral power after filtering out the MJO contribution. If the MJO contribution is retained, the bias becomes even more pronounced, as the MJO signal projects mostly on the symmetric part of the spectrum. The uncertainties in the PBE values were estimated using a chi-square test to obtain the confidence intervals of the variance to the $95 \%$ levels with 150 degrees of freedom as described in Gillard (2020). The mean uncertainty across all variables and all pressure levels in this figure is 0.009 . The power associated with the Kelvin and MRG + EIG0 waves (not shown) is biased toward the symmetric part of the spectrum across most variables and pressure levels, except for the meridional velocity in the upper troposphere and the vorticity, which are biased toward the antisymmetric part. This observation should be considered in conjunction with the meridional parity of the waves in the SW equations, where the zonal wind, the geopotential height and the divergence all have one parity (symmetric or antisymmetric) while the meridional wind and vorticity have the opposite parity. In addition, as we shall see in sections 4 and 5, the parity distribution of the simulated meridional wind and vorticity field in response to a small-scale stochastic forcing are also biased toward the antisymmetric part.

To understand the differences between the PBEs of the different dynamical variables we examined the individual power spectra in all 126 combinations of pressure levels and dynamical variables. A detailed discussion of the tropical wavenumber-frequency spectrum across all dynamical variables and pressure levels in reanalysis data is appropriate but is beyond the scope of the present work. Thus, we only mention two relevant observations. First, as noted by Gehne and Kleeman (2012), the geopotential height spectrum exhibit strong localized modes, which can be attribute to a barotropic Rossby-Haurwitz wave mode (Haurwitz 1940), a barotropic Kelvin wave mode localized around $k=1$ and $\omega \approx 0.75 \mathrm{cpd}$, and a barotropic MRG wave mode. The former two project on the symmetric part of the spectrum and the latter on the antisymmetric part. In addition the ERA5 geopotential height spectrum also exhibits localized modes corresponding to the spurious satellite modes identified by Wheeler and Kiladis (1999) in the OLR data. Second, in contrast to the red background characterizing all other variables, the meridional wind is characterized by a bimodal distribution in wavenumber, which is surprisingly similar to the midlatitude spectra observed in Sussman et al. (2020).

Before ending the ERA5 data discussion, a few more words are in order regarding the eastward-westward power distribution. As noted in Wheeler and Kiladis's analysis of satellite OLR observations, at least at high frequencies, there is more power in the westward-propagating part of the spectrum. In an analogy with the PBE defined in Eq. (2), we define the zonal bias estimator (ZBE) as follows:

$$
\mathrm{ZBE}=\frac{e-w}{e+w}
$$

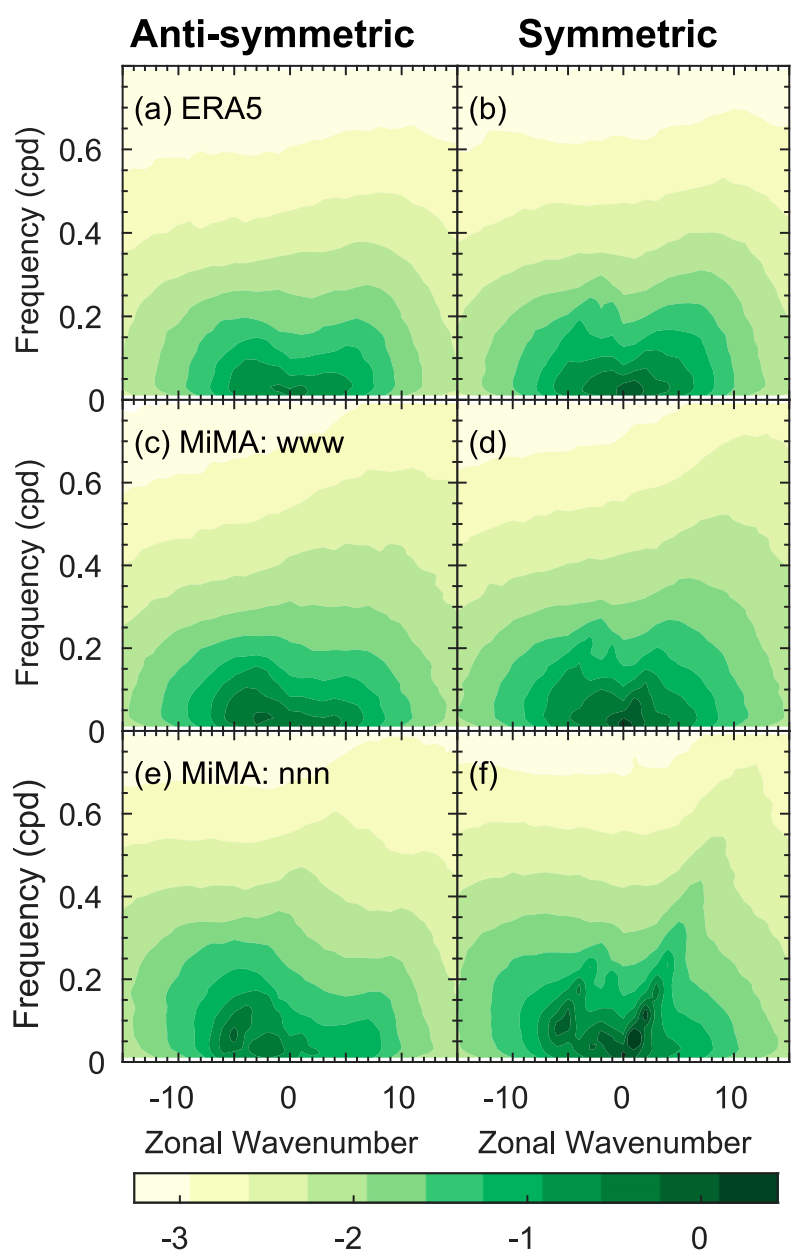

FIG. 4. Tropical wavenumber-frequency spectrum of the zonal wind at 300 mbar from (a),(b) ERA5 data, compared to (c),(d) the simulated spectrum in the MiMA model with topography, ocean heat fluxes, and land-sea contrast as described in section 2c (denoted by www in the figure), and (e),(f) without topography, ocean heat fluxes and land-sea contrast (denoted by nnn in the figure).

where $e$ and $w$ denote the total power in the eastward-propagating and westward-propagating parts of the wavenumber-frequency spectrum, respectively. The resulting ZBEs of the ERA5 data obtained using the total power over $k$ and $\omega$ are shown in Fig. 3 for the different pressure levels (columns) and different dynamical variables (rows). For comparison, the corresponding ZBE of the BT in Fig. 1 is $-0.05 \pm 0.01$. For the most part, across most pressure levels and most dynamical variables, the ZBE values in this figure are negative, corresponding to higher total power in the westward-propagating part of the spectra.

\section{The effects of large- and small-scale forcing on the parity distribution}

Having substantiated the existence of a symmetric bias in the tropical wavenumber-frequency spectrum, we now study the effects of both large- and small-scale forcing on its parity 


\section{(a) with topography; with ocean heat fluxes; with land-sea contrast}

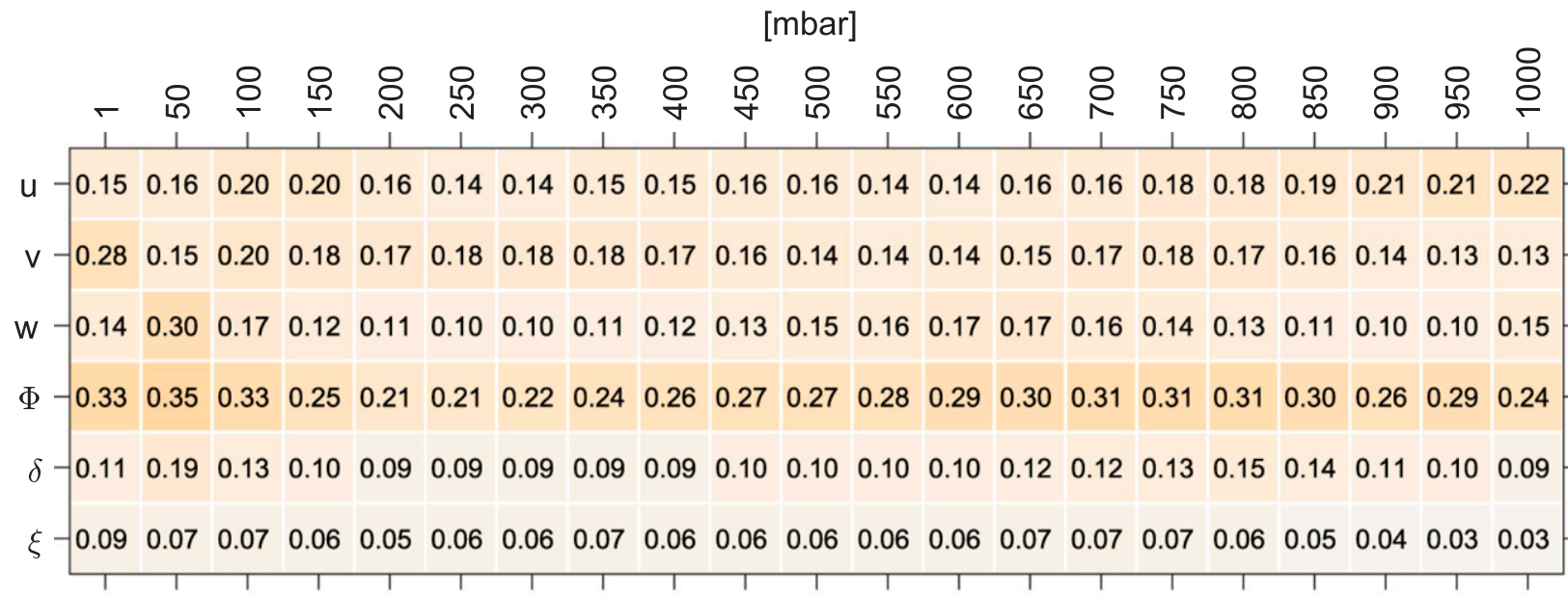

(b) no topography; no ocean heat fluxes; no land-sea contrast

$(\triangle P B E \leq 0.01)$

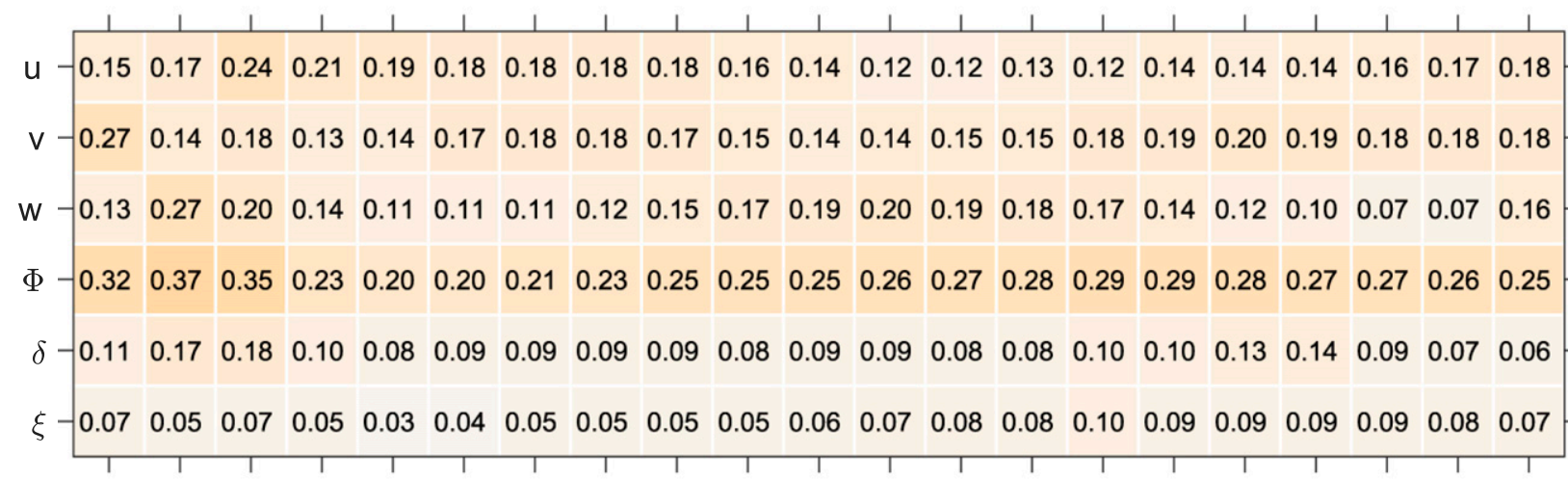

$-1$

FIG. 5. The PBE, defined in Eq. (2), in the tropical wavenumber-frequency spectra of the MiMA simulations (a) with and (b) without topography, ocean heat fluxes, and land-sea contrast for different pressure levels (columns) and each of the following dynamical variables (rows): zonal wind $(u)$, meridional wind $(v)$, vertical wind $\left(w\right.$, in $\left.\mathrm{Pa} \mathrm{s}^{-1}\right)$, geopotential height $(\Phi)$, divergence $(\delta)$, and vorticity $(\xi)$. The PBEs in this figure were estimated using the total power in the symmetric and antisymmetric components of the spectral peaks in the region $|k| \leq 15$ and $\omega \leq 0.8 \mathrm{cpd}$. The uncertainties in the PBEs were estimated using a chi-squared test to obtain the confidence intervals of the variance to the $95 \%$ levels with 150 degrees of freedom. The reported value at the top-right corner corresponds to the mean uncertainty across all variables and all pressure levels.

distribution. The tropical atmosphere is characterized by stationary waves and off-equatorial slowly varying convective maxima that induce meridional asymmetries on large spatial scales. We investigate the importance of these features for the parity distribution of the tropical wavenumber-frequency spectrum by considering the effects of large-scale forcing including topography, ocean heat fluxes, and land-sea contrast (i.e., the difference in heat capacity, surface friction, and moisture availability between oceans and continents). To this end we use an intermediate-complexity model of an idealized moist atmosphere (MiMA). The fidelity of the tropical wavenumber-frequency spectrum in the MiMA, compared to the ERA5 spectrum, is demonstrated in Fig. 4 using the zonal wind spectrum at 300 mbar. Despite a slightly more pronounced and more active Kelvin wave, the power spectrum in the model configuration with topography, with ocean heat fluxes, and with land-sea contrast (denoted by www) captures the observed power spectrum in the ERA5 data quite well. The power spectrum in the model configuration with no topography, no ocean heat fluxes, and no land-sea contrast (denoted by nnn) has an even more pronounced and more active Kelvin wave, as well as more active westward-propagating variability, compared to the ERA5 data. However, this configuration should not be expected to be comparable with observations. The particular model setup used in both cases follows Garfinkel et al. (2020b) and is briefly summarized in section 2c. It should be noted that Garfinkel et al. (2020b) configured the model to be representative of the Northern 
n-warmpool n-contrast n-topography
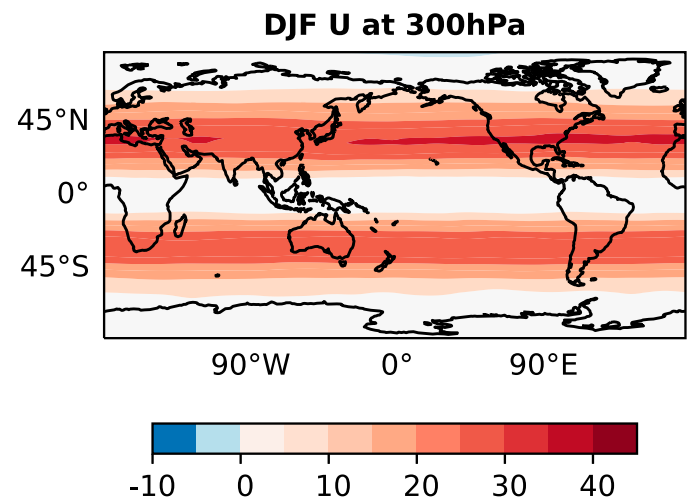

w-warmpool w-contrast w-topography

DJF $\mathrm{U}$ at $300 \mathrm{hPa}$
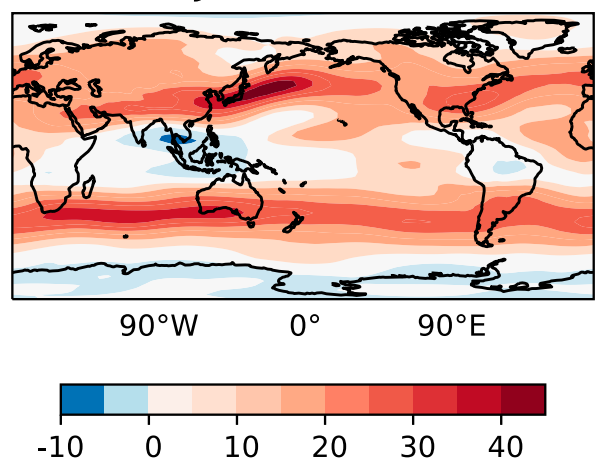

FIG. 6. Zonal wind at $300 \mathrm{mbar}$ during the Northern Hemisphere wintertime in the MiMA simulations (right) with and (left) without topography, ocean heat fluxes, and land-sea contrast.

Hemisphere wintertime (in terms of stationary waves), which is not necessarily representative on an annual mean. [The configuration in Garfinkel et al. (2020a) is representative of the annual mean, but the spectra are almost identical.] In addition, the model does not exhibit an MJO. Nevertheless, we argue that this model is useful for studying the parity bias in the tropical atmosphere (recall that the results of section 3 were obtained after filtering out the MJO contribution, which provides conservative estimates of the parity bias).

The resulting PBEs obtained using the total power for the MiMA simulations with and without topography, ocean heat fluxes, and land-sea contrast are shown in Fig. 5 for the different pressure levels (columns) and different dynamical variables (rows). Like the ERA5 data, the total power in this figure is biased toward the symmetric part of the spectrum across all pressure levels and dynamical variables. However, the main result of this figure is the similarity of the PBEs in the two experiments in Figs. 5a and 5b. Despite some noticeable differences between the two experiments, this figure implies that the parity distribution of the tropical wavenumberfrequency spectrum is only marginally sensitive to the largescale forcing induced by topography, ocean heat fluxes and land-sea contrast. Further appreciation of this result is obtained by considering the vast differences in the spatial structure of the winds during the Northern Hemisphere wintertime with and without topography, ocean heat fluxes, and land-sea contrast (Fig. 6). Note in particular the differences in the tropical winds, compared to the overall similar tropical spectra in Fig. 4.

While the PBEs in Fig. 5 are insensitive to the presence or absence of large-scale forcing, it is conceivable that the parity distribution of the wavenumber-frequency spectrum at large spatial scales is sensitive to small-scale forcing via upscale (inverse) turbulent energy cascade. Thus, we now investigate the effects of such a process on the parity distribution by adding a small-scale stochastic forcing, as detailed in section $2 \mathrm{e}$. The forcing is applied at wavenumbers $30 \leq|k| \leq 40$, near the limit of the spectral resolution of the model. While this choice of wavenumbers is not representative of small-scale convection in the tropics, it provides sufficient distance between the forcing and the response at low wavenumbers with $|k| \leq 10$ and enables us to examine the effects of the upscale energy cascade on the parity distribution. In addition, we apply this forcing to the MiMA configuration with no topography, ocean heat fluxes, and land-sea contrast in order to isolate the effects of the smallscale forcing from the large-scale ones. Figure 7 shows the resulting zonal wind spectrum at $300 \mathrm{mbar}$ in response to a purely symmetric forcing (Figs. 7a,b), a uniformly distributed forcing (Figs. 7c,d), and a purely antisymmetric forcing (Figs. 7e,f). Again, this model configuration should not be expected to be comparable with observations. Nevertheless, the total variance in the large-scale region of the wavenumberfrequency spectrum after adding the forcing is still in the ballpark of the observed tropical spectrum. For comparison, the color scale in this figure matches that of Fig. 4. In addition, the model captures the red background in both wavenumber and frequency, in the broad sense, and the spectral peaks are concentrated at the theoretically predicted linear wave modes (not shown).

In terms of the parity distribution, the response to an evenly distributed forcing in Fig. 7 appears to be nearly evenly distributed. In contrast, the responses to a purely symmetric forcing or purely antisymmetric forcing are noticeably biased toward the symmetric part of the spectrum. This suggests that the process of upscale turbulent energy cascade can affect the parity distribution at large spatial scales and that any asymmetry (symmetric or antisymmetric) leads to a symmetric bias. This idea is substantiated in Fig. 8, which shows the resulting PBEs obtained using the total power in the above three scenarios for the different pressure levels (columns) and different dynamical variables (rows). The purely symmetric forcing (Fig. 8a) and the purely antisymmetric forcing (Fig. 8c) have the same qualitative effect on the parity distribution. In both cases the tropical spectra of the zonal wind, vertical wind, geopotential height and divergence are all biased toward the symmetric part of the spectrum, while the tropical spectra of 

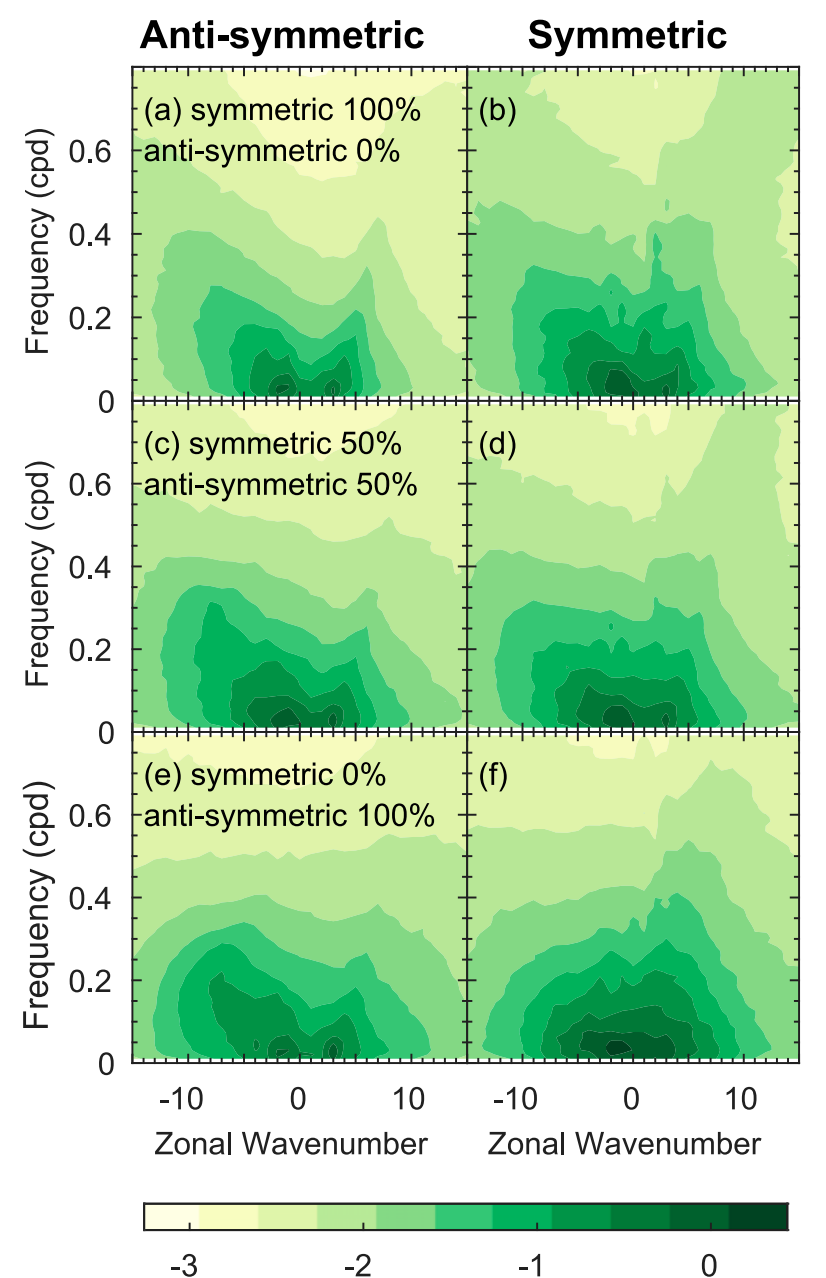

FIG. 7. Tropical wavenumber-frequency spectrum of the zonal wind at 300 mbar in the MiMA in response to the small-scale stochastic forcing in Eq. (1), with no topography, ocean heat fluxes, and land-sea contrast. (a),(b) In response to a purely symmetric forcing. (c),(d) In response to an evenly distributed forcing. (e),(f) In response to a purely antisymmetric forcing. The parity distribution of the forcing is controlled by masking in spectral space as detailed in section 2e. For the sake of comparison, the color scale in this figure is as in Fig. 4.

the meridional wind and vorticity are biased toward the antisymmetric part, at least in the troposphere. In contrast, the magnitudes of the PBEs in response to an evenly distributed forcing (Fig. 8b) are clearly lower than the former two.

Note, the PBEs of the vorticity and divergence fields in the lower troposphere in Fig. 8 are roughly opposite. In fact, the PBEs of the vorticity and divergence fields associated with the Kelvin and MRG+EIG0 waves (as opposed to the total power) are also roughly opposite (not shown). Thus, in what follows we focus on these two fields. Considering Helmholtz decomposition and the above results, this approach accounts for the parity distribution associated with both deep convection and incompressible motions. Note that the flow potential and streamfunction in a Helmholtz decomposition of the horizontal wind have the same parities as those of the divergence and vorticity fields, respectively.

Let us now examine the dependence of the parity distribution of the response on the parity distribution of the small-scale forcing in more details. To this end we let the parity of the input spectral energy vary from $100 \%$ symmetric to $100 \%$ antisymmetric with all consecutive decades in between for a total of 11 experiments as detailed in section 2e. The parity distribution of the forcing for the different experiments is illustrated in Fig. 9 using stacked bars to denote the amounts of symmetric (blue) and antisymmetric (orange) input spectral energies. The bar values in this figure are normalized such that the sum of the symmetric and antisymmetric components of the evenly distributed forcing (Exp 06) equals 1. This is done in order to emphasize that the input spectral energy across all experiments is held constant.

The resulting parity distribution as a function of the forcing parity in Fig. 9 is demonstrated in Fig. 10 using the divergence (Figs. 10a-c) and vorticity (Figs. 10d-f) fields at 850 mbar. As opposed to Fig. 9, the bar values in this figure are normalized such that the sum of the symmetric and antisymmetric components in each bar equals 1 , which guarantees that the symmetric and antisymmetric events are complementary. In the wavenumber region corresponding to the observed equatorial waves $|k| \leq 10$, the divergence field (Fig. 10a) is biased toward the symmetric part of the spectrum, except for Exp 06-09 where the response is evenly distributed. The vorticity field in this region (Fig. 10d) is biased toward the antisymmetric part of the spectrum, except for Exp 06 where the response is evenly distributed. As we shall see below, the bias in this region can be described as being quadratic in the forcing bias. In an attempt to understand the governing mechanism responsible for the formation of the parity bias we also examine, in the following section, the parity distribution in the wavenumber regions $10 \leq$ $|k| \leq 20$ (Figs. 10b,e) and $30 \leq|k| \leq 40$ (Figs. 10c,f). In the forcing region (Figs. 10c,f) the parity distribution of the divergence and vorticity fields spectra are consistent with the expected parities of zonally propagating wave solutions of the linearized forced-dissipated SW equations, where the divergence has the same parity as that of the forcing and the vorticity has an opposite parity. In the intermediate region, the parity distribution of the divergence field (Fig. 10b) varies from being biased toward the symmetric part in response to a symmetric forcing to unbiased in response to an antisymmetric forcing. The parity distribution of the vorticity field (Fig. 10e) varies from being biased toward the antisymmetric part in response to symmetric forcing to unbiased in response to moderately biased forcing to slightly biased toward the antisymmetric part in response to an antisymmetric forcing.

The above results demonstrate the effects of upscale turbulent energy cascade on the parity distribution of largescale wavenumber-frequency spectrum. In the following section we describe a mechanism that explains these results remarkably well.

\section{The effects of triad interactions}

The results obtained using the MiMA model in the previous section demonstrate the effects of upscale turbulent energy 
(a) $100 \%$ symmetric, $0 \%$ anti-symmetric

[mbar]

$(\triangle P B E \leq 0.02)$

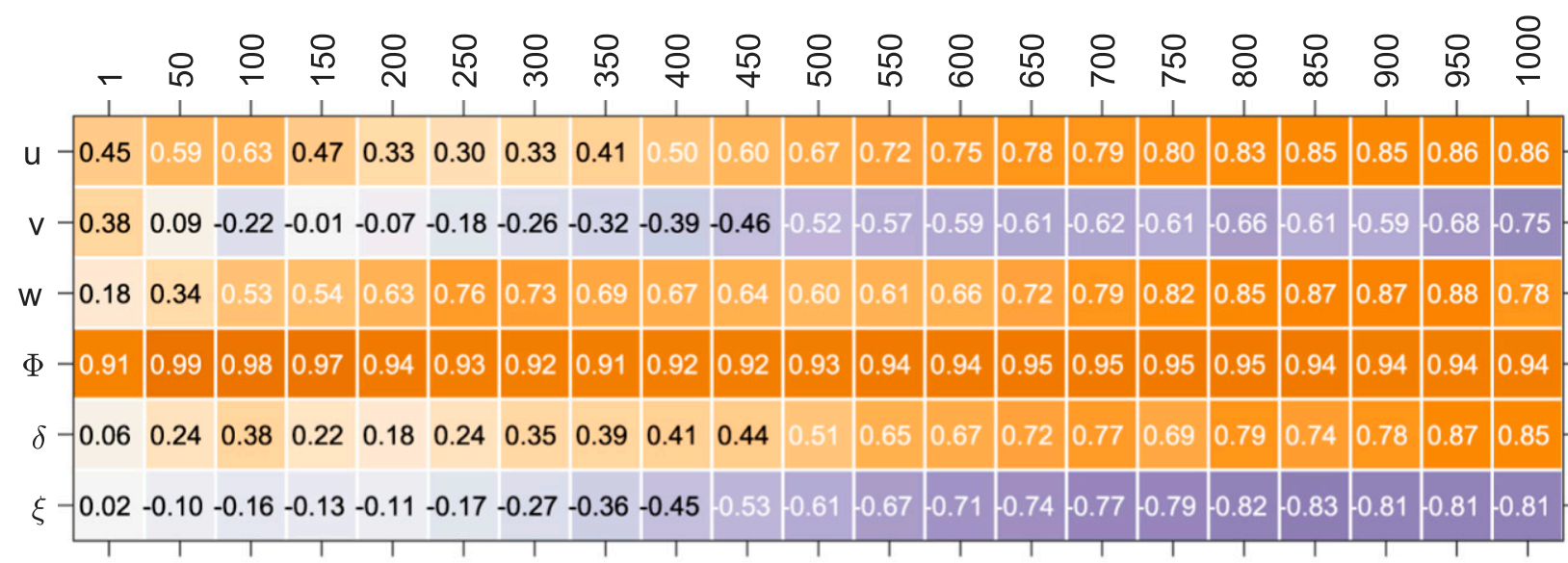

(b) $\mathbf{5 0} \%$ symmetric, $\mathbf{5 0 \%}$ anti-symmetric

$(\triangle P B E \leq 0.02)$

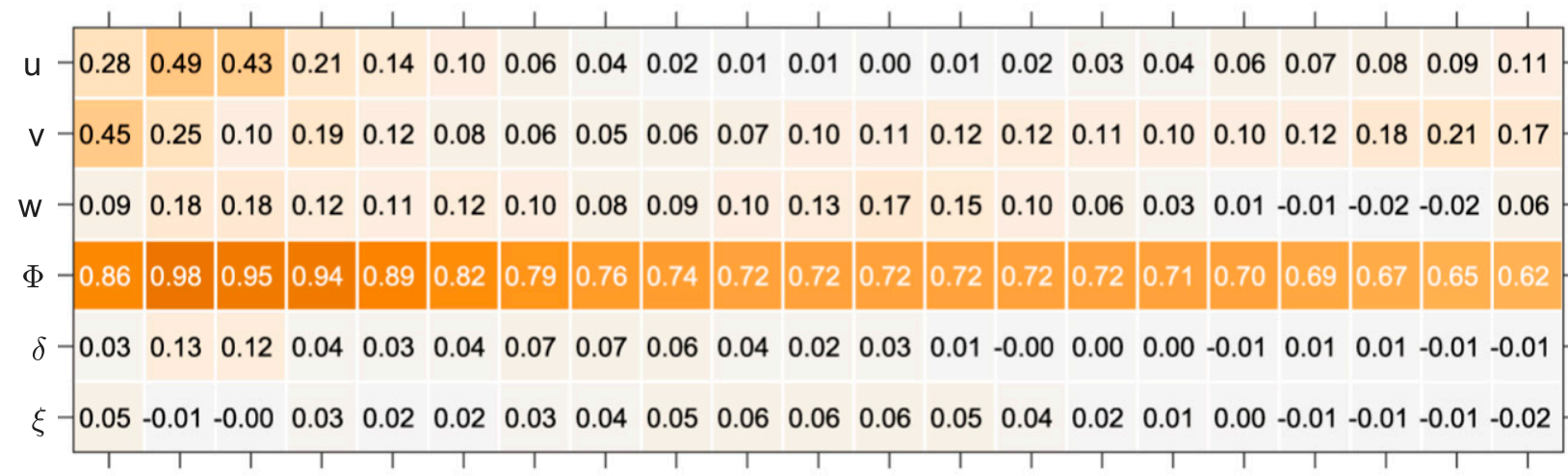

(c) $0 \%$ symmetric, $100 \%$ anti-symmetric

$(\triangle P B E \leq 0.02)$

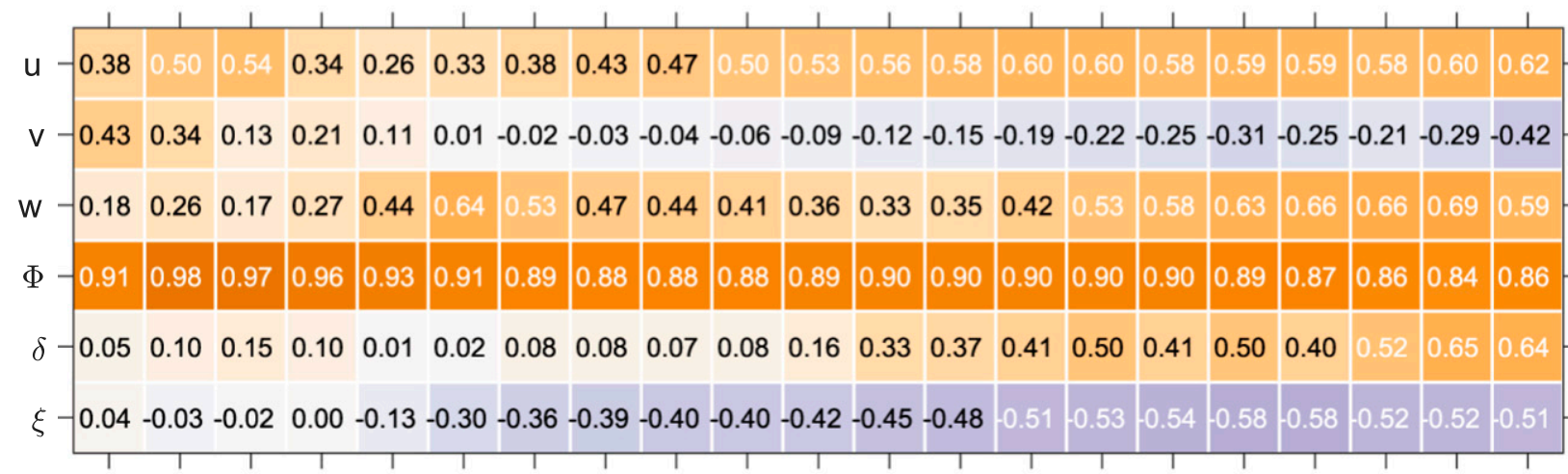

$-1$

0

1

FIG. 8. The PBE, defined in Eq. (2), in the tropical wavenumber-frequency spectra of the MiMA simulations in response to (a) a purely symmetric forcing, (b) an evenly distributed forcing, and (c) a purely antisymmetric forcing for different pressure levels (columns) and each of the following dynamical variables (rows): zonal wind $(u)$, meridional wind $(v)$, vertical wind $\left(w\right.$, in Pa s $\left.{ }^{-1}\right)$, geopotential height $(\Phi)$, divergence $(\delta)$, and vorticity $(\xi)$. The PBEs in this figure were estimated using the total power in the symmetric and antisymmetric components of the spectral peaks in the region $|k| \leq 15$ and $\omega \leq 0.8 \mathrm{cpd}$. The added small-scale stochastic forcing is given in Eq. (1). The uncertainties in the PBEs were estimated using a chi-squared test to obtain the confidence intervals of the variance to the $95 \%$ levels with 150 degrees of freedom. The reported value at the top-right corner corresponds to the mean uncertainty across all variables and all pressure levels. 


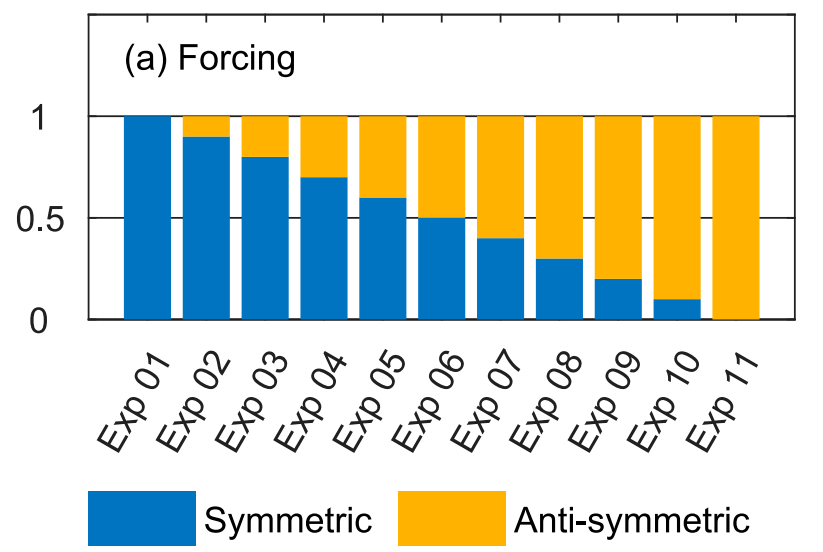

FIG. 9. Parity distribution of the applied forcing, in terms of the symmetric (blue) and antisymmetric (orange) input spectral energies. The bar values are normalized such that the sum of the symmetric and antisymmetric components of Exp 06 (evenly distributed forcing) equals 1 . The spectral weights applied in order to control the parity distribution of the forcing are detailed in section $2 \mathrm{~d}$ and Table 1 . The spectral weights were chosen so as to hold the amount of input spectral energy fixed between the different experiments.

cascade on the parity distribution of the wavenumber-frequency spectrum at certain pressure levels. While the results remain qualitatively similar at different pressure levels, the degree of the bias can vary. Thus, in order to eliminate this sensitivity, which is partly due to the imposed vertical structure of the forcing, we have repeated the experiments of Fig. 10 but in the framework of the forced dissipated SW model described in section $2 \mathrm{~d}$. The resulting parity distribution in the SW model subject to the forcing parity of Fig. 9 is shown in Fig. 11. Indeed, the biases in the large-scale $|k| \leq 10$ region (Figs. 11a,d), have a more pronounced quadratic form than those of the MiMA simulations shown in Fig. 10. In addition, the biases in the intermediate $10 \leq|k| \leq 20$ region (Figs. 11b,e) have a moderate quadratic form, excluding Exp 11.

The chosen wavenumber regions in Figs. 10 and 11 represent regions of possible triad interactions. Specifically, the region $|k| \leq 10$ corresponds to a region of possible triad interactions between waves in the forcing region $30 \leq|k| \leq 40$, while the region $10 \leq|k| \leq 20$ corresponds to possible triad interactions between waves in the region $|k| \leq 10$. Additional, independent, indication for the relevance of triad interactions in these regions is provided by Fig. 12 that shows the spectral power as a function of the forcing amplitude at wavenumbers: (Fig. 12a) $k=4$, (Fig. 12b) $k=14$, and (Fig. 12c) $k=34$ and frequency $\omega=0.19 \mathrm{cpd}$ (corresponding to the Kelvin wave at $k=4$ and equivalent depth of $50 \mathrm{~m}$ ). If two modes in the forcing region interact via quadratic nonlinearities, the power associated with the resulting wave will be the product of the power of each of the two constituent waves. Figure 12c shows that the power in the forcing region changes by two orders of magnitudes for every order of magnitude of the forcing amplitude (the slope of the best-fit curve is 2). Figure 12a shows that the power in the large-scale region changes by four orders of magnitude for every order of magnitude of the forcing amplitude (the slope of the best-fit curve is 4), which is indeed consistent with triad interactions in the forcing region. Slight changes to the sampled wavenumbers or frequencies lead to only small changes in the slopes. Likewise, the slope of the best-fit in Fig. 12b is close to 8, which is consistent with triad interactions between waves in the large-scale region. For more details on the contribution of wave-wave interactions in a moderately nonlinear turbulent flow to the background and wave spectrum the reader is referred to Garfinkel et al. (2021).

Let us now consider the effect of such triad interactions on the parity distribution. The longitude-time Fourier analysis decomposes the signal into essentially zonally propagating waves with latitude-dependent amplitudes. When two such waves interact via quadratic nonlinearities, they form a new zonally propagating wave whose latitude-dependent amplitude is the product of the latitude-dependent amplitudes of the former two. Thus, the parity of the resulting wave depends on the parity of the interacting waves. Let $P_{1}$ and $P_{2}$ denote the probabilities of waves 1 and 2 being symmetric (s) or antisymmetric (a). Then, assuming the waves have definite parities (so that the symmetric and antisymmetric events are complementary), the parity distribution of the resulting wave 3 is described by

$$
\begin{aligned}
& P_{3}(s)=P_{1}(s) P_{2}(s)+P_{1}(a) P_{2}(a), \\
& P_{3}(a)=P_{1}(a) P_{2}(s)+P_{1}(s) P_{2}(a) .
\end{aligned}
$$

The theoretical parity distribution of triad interactions according to Eq. (4) is shown in Fig. 13, in four relevant scenarios. The first scenario in Fig. 13a corresponds to interactions between waves distributed according to the parity distribution of the forcing in Fig. 9. This scenario, together with the observation of Fig. 12a that the power in the region $|k| \leq 10$ results from triad interactions of waves in the forcing region, and together with the observation of Fig. 11c that the parity distribution of the divergence field spectrum in the forcing region is the same as the forcing parity, explain the observed quadratic form of the divergence field spectrum in Fig. 11a.

The second scenario in Fig. 13b corresponds to interactions between waves distributed according to the parity distribution of the forcing in Fig. 9 and waves with an opposite parity distribution. Again, this scenario, together with the observation of Fig. 12a that the power in the region $|k| \leq 10$ results from triad interactions of waves in the forcing region, and together with the observation of Fig. $11 \mathrm{f}$ that the parity distribution of the vorticity field spectrum in the forcing region is opposite to the forcing parity, explain the observed inverse quadratic form of the vorticity field spectrum in Fig. 11d.

The third scenario in Fig. 13c corresponds to interactions between waves distributed according to Fig. 13a. The theoretically expected parity distribution of the new wave in this scenario is a more moderate quadratic form. Despite some noticeable differences, the observed parity distribution of the 


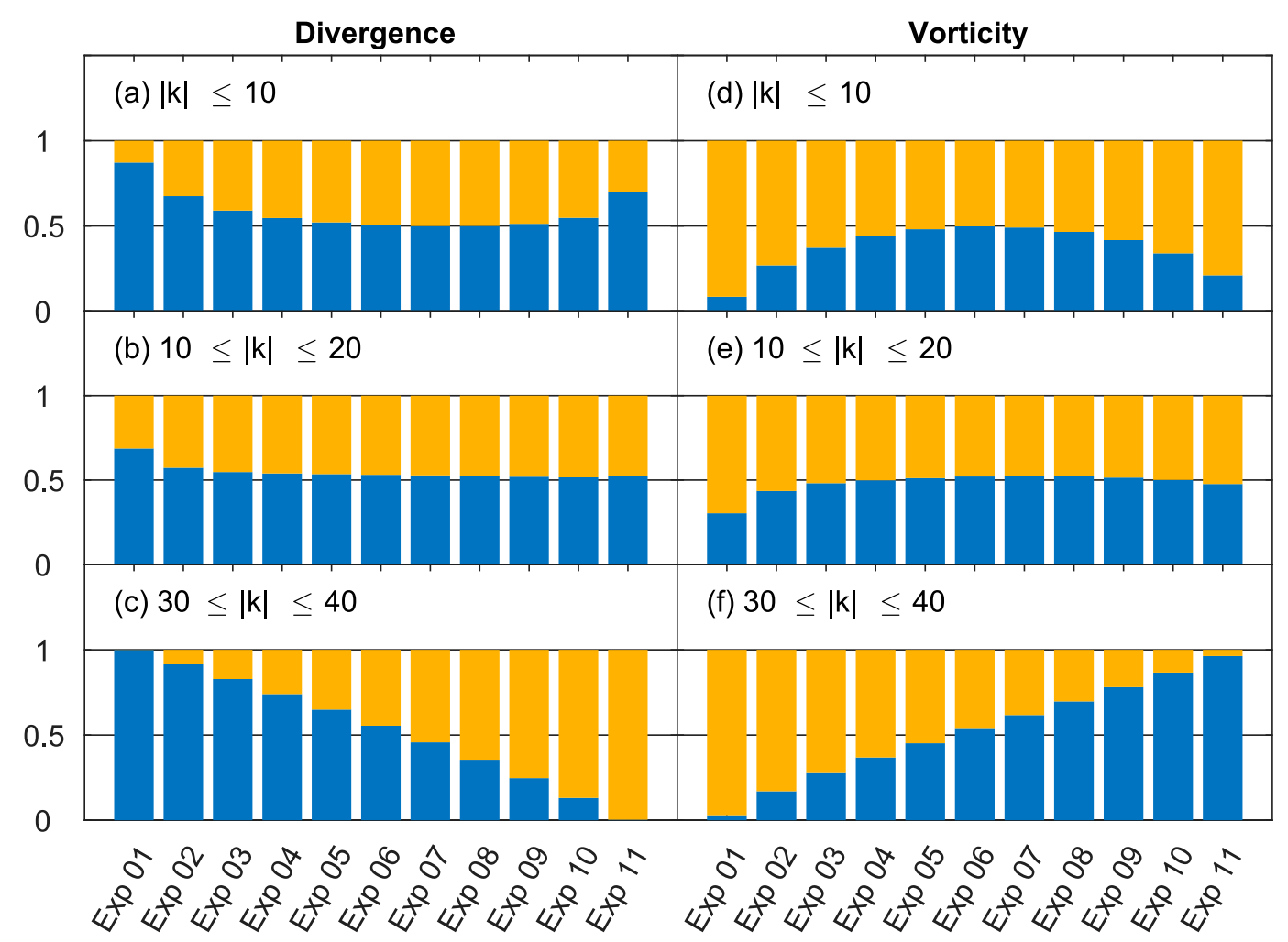

Symmetric

Anti-symmetric

FIG. 10. Parity distribution of the tropical wavenumber-frequency spectra of the (left) divergence and (right) vorticity fields simulated using the MiMA. The parity distribution in this figure is estimated using the total power contained in the symmetric (blue) and antisymmetric (orange) parts of the spectrum within (a),(d) the large-scale region $|k| \leq 10$, (b),(e) intermediate region $10 \leq|k| \leq 20$, and (c),(f) the forcing region $30 \leq|k| \leq 40$. In all cases, the total power is taken over $\omega \leq 0.8 \mathrm{cpd}$. The bar values in each panel are normalized such that the sum of the symmetric and antisymmetric components equals 1 .

divergence in Fig. 11b does correspond to a more moderate quadratic form (compared to Fig. 11a), excluding Exp 11. Hence, this scenario, together with the observation of Fig. 12b that the power in the region $10 \leq|k| \leq 20$ results from triad interactions of waves in the region $|k| \leq 10$, qualitatively explain the observed parity distribution in Fig. 11b. Using similar reasoning explains the observed parity distribution in Fig. 11e as the result of triad interactions between waves distributed according to Fig. 11d.

The fourth scenario in Fig. 13d corresponds to the average of interactions involving waves in Fig. 13b with the forcing and interaction involving waves in Fig. 13b with waves in Fig. 13a, i.e., interactions that couple the divergence and vorticity fields. This combination was found by trial and error and accurately explains the observed parity distribution in Fig. 10e. Thus, the proposed mechanism can explain the observed parity distribution in Fig. 10e as well, but the identification of the relevant waves is not straightforward in that case.

The above mechanism explains the parity distribution in Figs. 10 and 11 remarkably well. However, its applicability to the observed parity distribution in the tropical atmosphere is more intricate for two reasons: First, the observed parity bias is less pronounced and noisier than in the MiMA and SW model simulations. Second, while the total variance in the large-scale region of the wavenumber-frequency spectrum after adding the forcing remains in the ballpark of the observed tropical spectrum, the applied forcing does, indeed, introduce superfluous variability not present in observations. Thus, the proposed mechanism can only provide a qualitative explanation for the observed symmetric bias. In addition, as noted in sections $2 \mathrm{e}$ and 4 , the applied forcing is not representative of small-scale convection in the tropics, which has typical wavenumbers in the range $1000 \leqq|k| \lesssim 4000$. Nevertheless, our mechanism describes the effects of upscale turbulent energy cascade on the parity distribution, the process by which small-scale convection triggers large-scale features in the tropics. In this regard, another noteworthy point is the fact that the classical theory of fully developed turbulence by Kolmogorov (1991) applies to isotropic (and homogeneous) turbulence, where the notion of parity of the waves involved in triad interactions is meaningless. The relevance of moderate turbulence, as opposed to fully developed turbulence, for variability in the tropical atmosphere is also studied in Garfinkel et al. (2021). With these caveats in mind, the proposed mechanism sheds light on the 


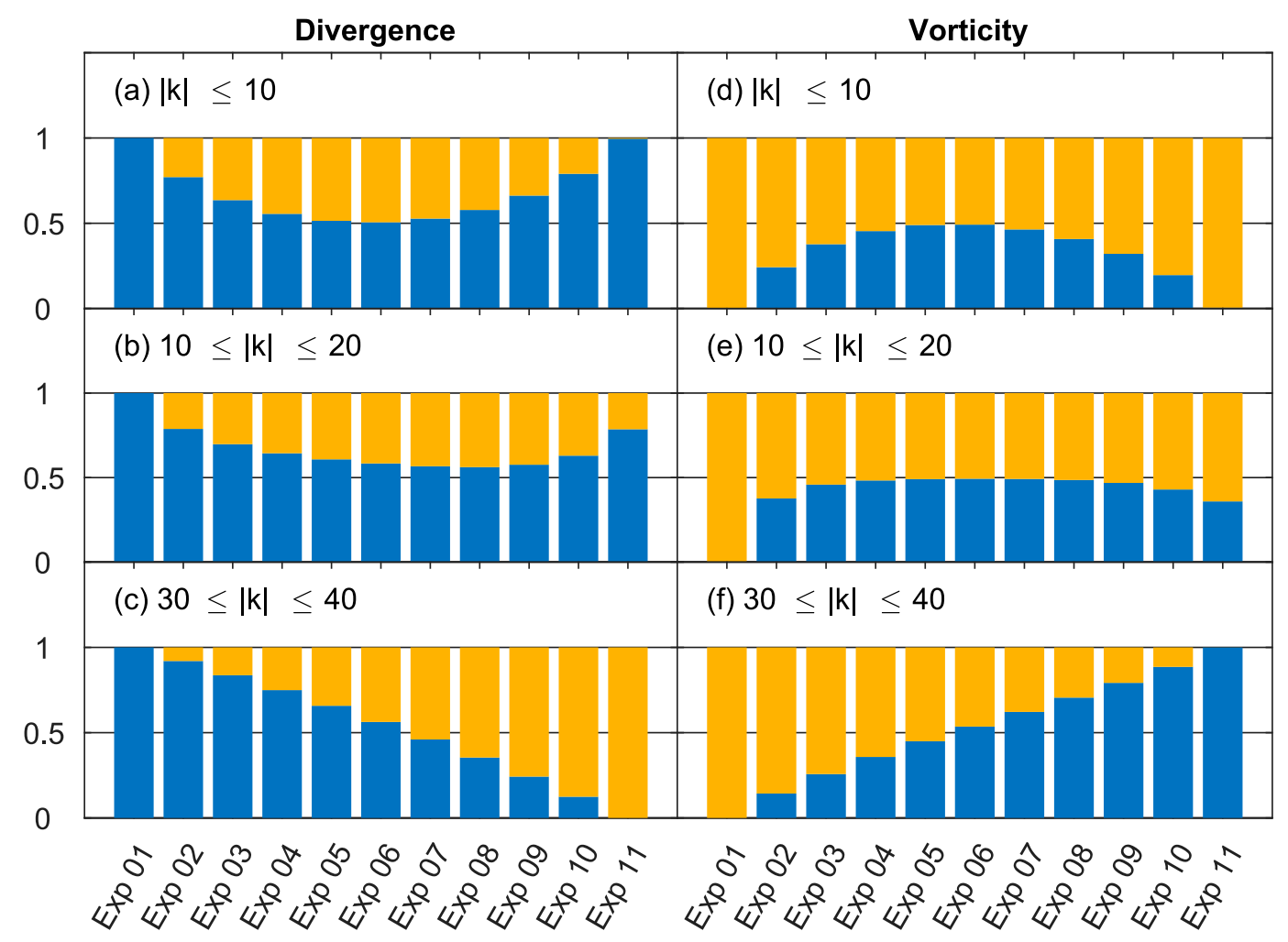

Symmetric

Anti-symmetric

FIG. 11. As in Fig. 10, but for the simulated (left) divergence and (right) vorticity fields in the SW model instead of the MiMA model.

effects of triad interactions on the parity distribution, and may be relevant to the observed parity distribution in the tropical atmosphere.

\section{Summary and discussion}

Satellite-derived observations indicate that the tropical wavenumber-frequency spectrum of deep-convection proxies is biased toward the symmetric component of the spectrum. The degree of the bias in terms of the total power in the observed brightness temperature (BT) spectrum is between $20 \%$ and $30 \%$ (depending on MJO filtering), relative to the antisymmetric component. More evidence for the symmetric bias in the tropical spectrum is found in reanalysis data, where the zonal wind, meridional wind, vertical wind, geopotential, divergence, and vorticity fields are all biased toward the symmetric part of the spectrum to some degree all the way from the surface to the upper stratosphere/lower mesosphere. These observations bring about the notion of the parity distribution of the tropical spectrum, i.e., the distribution of power between its symmetric and antisymmetric components.

The ubiquity of the symmetric bias in reanalysis data motivates a mechanistic study of the parity distribution in the tropics. To this end we have studied the parity distribution's sensitivity to both large- and small-scale forcing. Using an intermediate-complexity model of an idealized moist atmosphere, we find that the parity distribution of the wavenumberfrequency spectrum is nearly insensitive to the large-scale meridional asymmetries induced by topography, ocean heat fluxes, and land-sea contrast. On the other hand, we find that a small-scale (stochastic) forcing has the capacity to affect the parity distribution at large spatial scales via the process of upscale (inverse) turbulent energy cascade. Based on the results of the present study, a possible mechanism for explaining some of the symmetric bias in the wavenumberfrequency spectrum is that of triad interactions. When two zonally propagating waves interact via quadratic nonlinearities, they form a new zonally propagating wave whose latitude-dependent amplitude is the product of the former two. Thus, once an asymmetry (symmetric or antisymmetric) is formed in the parity distribution at small spatial scales, waves are more likely to interact with same-parity waves, thereby generating large-scale symmetric waves.

Physically, some of the large-scale features in the tropical spectrum are believed to be excited by small-scale convection via an upscale turbulent energy cascade. While the applied forcing used in the present work is not representative of the small-scale convection in the tropics, it provides sufficient distance between the forcing and the response to capture the effects of upscale energy cascade on the parity distribution. 

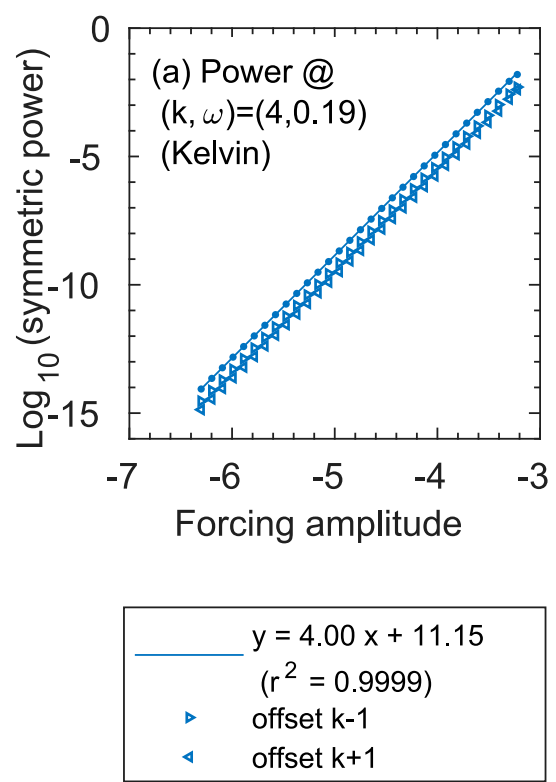
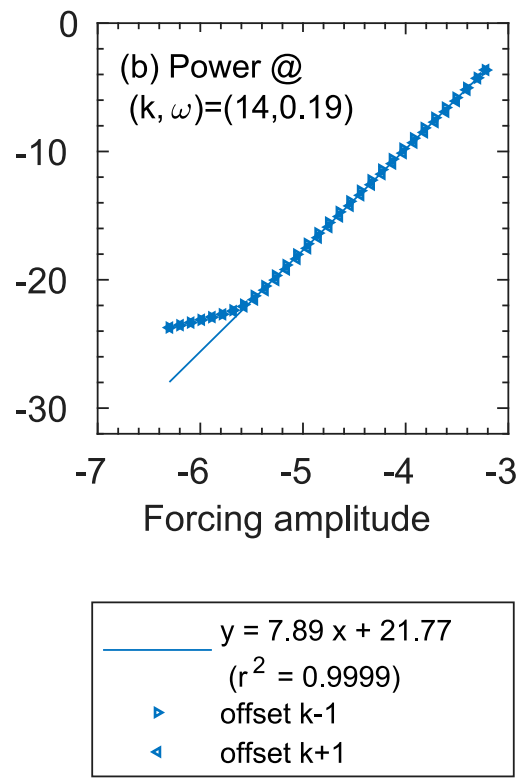

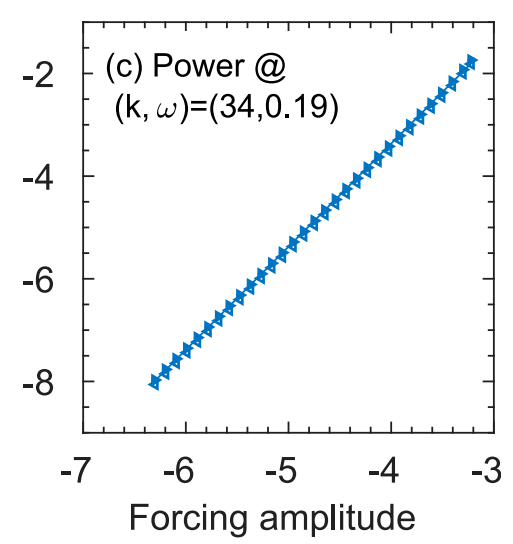

\begin{tabular}{ll}
\hline & $y=2.01 \mathrm{x}+4.67$ \\
& $\left(\mathrm{r}^{2}=0.9999\right)$ \\
& offset $\mathrm{k}-1$ \\
$\triangleleft \quad$ & offset $\mathrm{k}+1$
\end{tabular}

FIG. 12. Spectral power as a function of the forcing amplitude at wavenumbers (a) $k=4$, (b) $k=14$, and (c) $k=34$ and frequency $\omega=$ $0.19 \mathrm{cpd}$ (corresponding to the Kelvin wave at $k=4$ and equivalent depth of $50 \mathrm{~m}$ ). In all cases the power is also sampled at neighboring wavenumbers with the same value of $\omega$ (outside the waves' dispersion curve). Similar results are obtained for $\omega=0.1,0.3$, and 0.5 . The results of the antisymmetric EIG0 wave are similar to those of the Kelvin wave in (a). The abscissas in all panels change by four orders of magnitude. The ordinates in (a)-(c) change by 16, 32, and 8 orders of magnitude, respectively, so the 1:1 lines correspond to slopes of 4,8 , and 2 , respectively.

Thus, the results of the present study demonstrate that the physical process associated with deep convection leads to a symmetric bias in the parity distribution of the tropical spectrum. In a recent work, Garfinkel et al. (2021) showed that the observed combination of large-scale background and wave spectrum can be excited by small-scale forcing in a moderately nonlinear turbulent flow. However, a thorough description of this process is still lacking.

The results of the present study and their possible implications to the role of deep convection in the tropics emphasize the role played by nonlinear processes in the formation of the tropical wavenumber-frequency spectrum. In particular, the response to purely antisymmetric forcing is purely symmetric, which is in contrast to the linear model used in Salby and Garcia (1987) where purely antisymmetric forcing can only trigger purely antisymmetric waves.

In addition to the variability generated in situ by deep convection, much of the large-scale tropical variability is also attributed to extratropical sources (see, e.g., Stan et al. 2017; Straub and Kiladis 2003, and references therein). A proper description of the parity distribution and symmetric bias in the tropical spectrum should address these sources as well. Unfortunately, attempting to quantify the contributions of different sources to the parity distribution is challenging for three reasons. First, a quantitative description of the relative contributions of deep convection and extratropical forcing is still lacking. Second, they are not independent as the latter also forces deep convection in the tropics. Third, their effect on the parity distribution is intricate. For example, the latter may project on the tropical wavenumber-frequency spectrum directly via Rossby wave trains that enter the tropics with a typical period of several days and a typical wavenumber $\sim 6$, which induces antisymmetric variability, but also indirectly by exciting Kelvin waves, which induces symmetric variability. Thus, the present work only demonstrates the effects of smallscale forcing on the parity distribution.

In the opposite direction, the tropics also influence the extratropics, and hence the parity distribution of the tropical wavenumber-frequency spectrum has possible implications for the extratropics. Once the tropical wave modes are forced, they can propagate both vertically and meridionally and affect the large-scale circulation. The remote response should maintain the parity distribution of the forcing, and hence the regions of the midlatitudes with say, a low versus a high will depend on the parity of the wave in the tropics. For example, in the classical Gill response (Gill 1980) there is a symmetric, offequatorial Rossby wave response to the west of the heating. These ridges then launch wave trains to midlatitudes (Hoskins and Karoly 1981). If the tropical response was antisymmetric instead, then the phasing of the extratropical response would also differ. For example, the greater importance of symmetric modes is crucial for understanding why, e.g., El Niño has teleconnections with the phasing that is observed.

In conclusion, the power distribution between the symmetric and antisymmetric components of the tropical wavenumberfrequency spectrum may be used to shed light on the role of different processes in the tropics. In the present work we have demonstrated the effects of upscale energy cascade, which is relevant to deep convection, on the symmetric bias, but other processes can play a role as well. We hope that the 


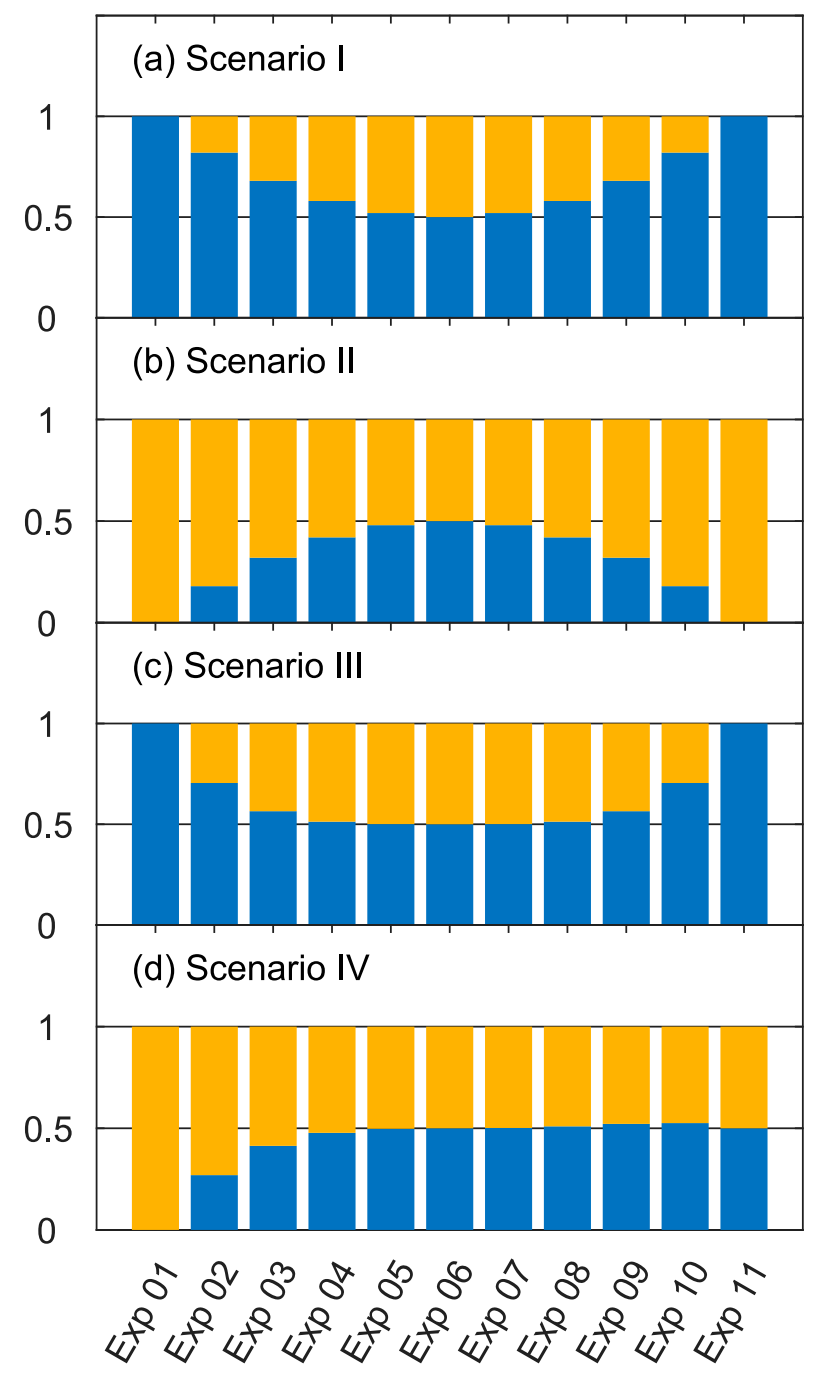

Symmetric

FIG. 13. Theoretical parity distribution calculated using Eq. (4) for the following four scenarios: (a) Triad interactions involving waves distributed according to the applied forcing in Fig. 9. (b) Triad interactions involving waves distributed according to the applied forcing and waves with an opposite distribution. (c) Triad interactions involving waves distributed according to (a) of this figure. (d) The average of triad interactions involving waves in (b) with the forcing and triad interaction involving waves in (b) with waves in (a).

present study will motivate complementary phenomenological studies by the community, aimed at quantifying the contributions of particular sources of variability to the parity distribution.

Acknowledgments. Brightness temperature data were downloaded from the CLAUS archive held at the British Atmospheric Data Centre, produced using ISCCP source data distributed by the NASA Langley Data Center. We acknowledge the support of a European Research Council starting grant under the EU Horizon 2020 research and innovation programme (Grant Agreement 677756). We thank the three anonymous reviewers for their constructive comments and specifically a particular one for pointing out the bug in the NCAR Command Language routine used for calculating the spectra.

\section{REFERENCES}

Adames, Á. F., and D. Kim, 2016: The MJO as a dispersive, convectively coupled moisture wave: Theory and observations. J. Atmos. Sci., 73, 913-941, https://doi.org/10.1175/JAS-D-150170.1.

Barnes, E. A., D. L. Hartmann, D. M. Frierson, and J. Kidston, 2010: Effect of latitude on the persistence of eddy-driven jets. Geophys. Res. Lett., 37, L11804, https://doi.org/10.1029/ 2010 GL043199.

Biello, J. A., and A. J. Majda, 2005: A new multiscale model for the Madden-Julian oscillation. J. Atmos. Sci., 62, 1694-1721, https://doi.org/10.1175/JAS3455.1.

Boyd, J. P., 2001: Chebyshev and Fourier Spectral Methods. Courier Corporation, $668 \mathrm{pp}$.

Chao, W. C., 1987: On the origin of the tropical intraseasonal oscillation. J. Atmos. Sci., 44, 1940-1949, https://doi.org/10.1175/ 1520-0469(1987)044<1940:OTOOTT>2.0.CO;2.

Cho, H.-K., K. P. Bowman, and G. R. North, 2004: Equatorial waves including the Madden-Julian oscillation in TRMM rainfall and OLR data. J. Climate, 17, 4387-4406, https:// doi.org/10.1175/3215.1.

Delplace, P., J. Marston, and A. Venaille, 2017: Topological origin of equatorial waves. Science, 358, 1075-1077, https://doi.org/ 10.1126/science.aan8819.

Garfinkel, C. I., I. Fouxon, O. Shamir, and N. Paldor, 2017: Classification of eastward propagating waves on the spherical Earth. Quart. J. Roy. Meteor. Soc., 143, 1554-1564, https:// doi.org/10.1002/qj.3025.

— I. White, E. P. Gerber, and M. Jucker, 2020a: The impact of SST biases in the tropical east Pacific and Agulhas Current region on atmospheric stationary waves in the Southern Hemisphere. J. Climate, 33, 9351-9374, https://doi.org/10.1175/ JCLI-D-20-0195.1.

$-,-,-\longrightarrow, \ldots$, and M. Erez, 2020b: The building blocks of Northern Hemisphere wintertime stationary waves. J. Climate, 33, 5611-5633, https://doi.org/10.1175/JCLI-D19-0181.1.

— O. Shamir, I. Fouxon, and N. Paldor, 2021: Tropical background and wave spectra: Contribution of wave-wave interactions in a moderately nonlinear turbulent flow. J. Atmos. Sci., https://doi.org/10.1175/JAS-D-20-0284.1, 78, 1773-1789.

Gehne, M., and R. Kleeman, 2012: Spectral analysis of tropical atmospheric dynamical variables using a linear shallow-water modal decomposition. J. Atmos. Sci., 69, 2300-2316, https:// doi.org/10.1175/JAS-D-10-05008.1.

Gill, A. E., 1980: Some simple solutions for heat-induced tropical circulation. Quart. J. Roy. Meteor. Soc., 106, 447-462, https:// doi.org/10.1002/qj.49710644905.

Gillard, J., 2020: A First Course in Statistical Inference. Springer, $164 \mathrm{pp}$.

Haurwitz, B., 1940: The motion of atmospheric disturbances on the spherical Earth. J. Mar. Res., 3, 254-267.

Hendon, H. H., and M. C. Wheeler, 2008: Some space-time spectral analyses of tropical convection and planetary-scale waves. J. Atmos. Sci., 65, 2936-2948, https://doi.org/10.1175/ 2008JAS2675.1. 
Hersbach, H., and Coauthors, 2020: The ERA5 global reanalysis. Quart. J. Roy. Meteor. Soc., 146, 1999-2049, https://doi.org/ 10.1002/qj.3803.

Hodges, K., D. Chappell, G. Robinson, and G. Yang, 2000: An improved algorithm for generating global window brightness temperatures from multiple satellite infrared imagery. J. Atmos. Oceanic Technol., 17, 1296-1312, https://doi.org/10.1175/15200426(2000)017<1296:AIAFGG > 2.0.CO;2.

Hoskins, B. J., and D. J. Karoly, 1981: The steady linear response of a spherical atmosphere to thermal and orographic forcing. J. Atmos. Sci., 38, 1179-1196, https://doi.org/10.1175/15200469(1981)038<1179:TSLROA > 2.0.CO;2.

Jucker, M., and E. Gerber, 2017: Untangling the annual cycle of the tropical tropopause layer with an idealized moist model. J. Climate, 30, 7339-7358, https://doi.org/10.1175/JCLI-D-170127.1.

Kacimi, A., and B. Khouider, 2018: The transient response to an equatorial heat source and its convergence to steady state: Implications for MJO theory. Climate Dyn., 50, 3315-3330, https://doi.org/10.1007/s00382-017-3807-6.

Kiladis, G. N., M. C. Wheeler, P. T. Haertel, K. H. Straub, and P. E. Roundy, 2009: Convectively coupled equatorial waves. Rev. Geophys., 47, RG2003, https://doi.org/10.1029/2008RG000266.

Kolmogorov, A. N., 1991: The local structure of turbulence in incompressible viscous fluid for very large Reynolds numbers. Proc. Roy. Soc. London, 434A, 9-13, https://doi.org/10.1098/ rspa.1991.0075.

Majda, A. J., and S. N. Stechmann, 2009: The skeleton of tropical intraseasonal oscillations. Proc. Natl. Acad. Sci. USA, 106, 8417-8422, https://doi.org/10.1073/pnas.0903367106.

Masunaga, H., T. S. L'Ecuyer, and C. D. Kummerow, 2006: The Madden-Julian oscillation recorded in early observations from the Tropical Rainfall Measuring Mission (TRMM). J. Atmos. Sci., 63, 2777-2794, https://doi.org/10.1175/JAS3783.1.

Matsuno, T., 1966: Quasi-geostrophic motions in the equatorial area. J. Meteor. Soc. Japan, 44, 25-43, https://doi.org/10.2151/ jmsj1965.44.1_25.
Orlanski, I., and L. J. Polinsky, 1977: Spectral distribution of cloud cover over Africa. J. Meteor. Soc. Japan, 55, 483-493, https:// doi.org/10.2151/jmsj1965.55.5_483.

Paldor, N., I. Fouxon, O. Shamir, and C. I. Garfinkel, 2018: The mixed Rossby-gravity wave on the spherical Earth. Quart. J. Roy. Meteor. Soc., 144, 1820-1830, https://doi.org/10.1002/ qj.3354.

Salby, M. L., and R. R. Garcia, 1987: Transient response to localized episodic heating in the tropics. Part I: Excitation and short-time near-field behavior. J. Atmos. Sci., 44, 458-498, https://doi.org/ 10.1175/1520-0469(1987)044<0458:TRTLEH>2.0.CO;2.

Sobel, A., and E. Maloney, 2012: An idealized semi-empirical framework for modeling the Madden-Julian oscillation. J. Atmos. Sci., 69, 1691-1705, https://doi.org/10.1175/JASD-11-0118.1.

Stan, C., D. M. Straus, J. S. Frederiksen, H. Lin, E. D. Maloney, and C. Schumacher, 2017: Review of tropical-extratropical teleconnections on intraseasonal time scales. Rev. Geophys., 55, 902-937, https://doi.org/10.1002/2016RG000538.

Straub, K. H., and G. N. Kiladis, 2003: Extratropical forcing of convectively coupled Kelvin waves during austral winter. J. Atmos. Sci., 60, 526-543, https://doi.org/10.1175/15200469(2003)060<0526:EFOCCK >2.0.CO;2.

Sussman, H. S., A. Raghavendra, P. E. Roundy, and A. Dai, 2020: Trends in northern midlatitude atmospheric wave power from 1950 to 2099. Climate Dyn., 54, 2903-2918, https://doi.org/ 10.1007/s00382-020-05143-3.

Vallis, G. K., E. P. Gerber, P. J. Kushner, and B. A. Cash, 2004: A mechanism and simple dynamical model of the North Atlantic Oscillation and annular modes. J. Atmos. Sci., 61, 264-280, https://doi.org/10.1175/1520-0469(2004)061<0264:AMASDM> 2.0.CO;2.

Wheeler, M., and G. N. Kiladis, 1999: Convectively coupled equatorial waves: Analysis of clouds and temperature in the wavenumber-frequency domain. J. Atmos. Sci., 56, 374-399, https://doi.org/10.1175/1520-0469(1999)056<0374:CCEWAO> 2.0.CO;2. 